\title{
Absence of Face-specific Cortical Activity in the Complete Absence of Awareness: Converging Evidence from Functional Magnetic Resonance Imaging and Event-related Potentials
}

\author{
Valia Rodríguez ${ }^{1 *}$, Russell Thompson ${ }^{2}$, Mark Stokes ${ }^{3}$, Matthew Brett ${ }^{4}$, \\ Indira Alvarez ${ }^{1}$, Mitchell Valdes-Sosa ${ }^{1}$, and John Duncan ${ }^{2 *}$
}

\begin{abstract}
In this study, we explored the neural correlates of perceptual awareness during a masked face detection task. To assess awareness more precisely than in previous studies, participants employed a 4-point scale to rate subjective visibility. An eventrelated fMRI and a high-density ERP study were carried out. Imaging data showed that conscious face detection was linked to activation of fusiform and occipital face areas. Frontal and parietal regions, including the pre-SMA, inferior frontal sulcus, anterior insula/frontal operculum, and intraparietal sulcus, also responded strongly when faces were consciously perceived. In contrast, no brain area showed face-selective activity when
\end{abstract}

\section{INTRODUCTION}

The role played by different cortical areas in generating conscious perception is the subject of much debate, but the observation that activity can occur in sensory processing areas in the apparent absence of conscious perception has been replicated using several different paradigms. For example, activation of face-specific and house-specific areas has been observed for faces and houses that were not seen during a binocular color fusion experiment (Moutoussis \& Zeki, 2002). Similarly, object images that were suppressed by interocular competition elicited the activation of posterior dorsal cortical regions (Fang \& He, 2005). Activity has also been observed in ventral visual areas in response to masked words that cannot be explicitly reported (Dehaene et al., 2001) and during change detection studies on trials where participants were blind to the change (Pessoa \& Ungerleider, 2004; Beck, Rees, Frith, \& Lavie, 2001).

Evidence that unseen stimuli undergo some kind of unconscious processing in object-selective visual areas has been taken to imply that such activity is not sufficient

${ }^{1}$ Cuban Neuroscience Center, ${ }^{2}$ Medical Research Council Cognition and Brain Sciences Unit, Cambridge, United Kingdom,

${ }^{3}$ University of Oxford, ${ }^{4}$ University of California-Berkeley

*These authors contributed equally to this study. participants reported no impression of a face. ERP results showed that conscious face detection was associated with enhanced N170 and also with the presence of a second negativity around $300 \mathrm{msec}$ and a slow positivity around $415 \mathrm{msec}$. Again, face-related activity was absent when faces were not consciously perceived. We suggest that, under conditions of backward masking, ventral stream and fronto-parietal regions show similar, strong links of face-related activity to conscious perception and stress the importance of a detailed assessment of awareness to examine activity related to unseen stimulus events.

for conscious access. It has been proposed that access to consciousness is associated with the additional involvement of fronto-parietal areas as a top-down amplification network directed toward perceptual regions (Baars, 2002; Dehaene \& Naccache, 2001). Support for this proposal comes from a variety of sources, including studies of seen and unseen changes in a visual scene (Pessoa \& Ungerleider, 2004; Beck et al., 2001), of perceptual transitions in binocular rivalry (Lumer, Friston, \& Rees, 1998) and of bistable perception (Kleinschmidt, Buchel, Zeki, \& Frackowiak, 1998). Contradicting the proposal are recent studies that have found activation in areas belonging to the frontoparietal network in relation to the stimuli that are reported as not consciously perceived. Attentional blink studies, for example, have found activity linked to missed targets in some frontal and parietal areas (Shapiro, Johnston, Vogels, Zaman, \& Roberts, 2007; Kranczioch, Debener, Schwarzbach, Goebel, \& Engel, 2005). A similar result was recently reported in a study of implicit processing where activation of middorsolateral $\mathrm{pFC}$ was found associated with subliminal prime perception (Lau \& Passingham, 2007).

A small handful of studies (Sterzer, Jalkanen, \& Rees, 2009; Sterzer, Haynes, \& Rees, 2008; Fang \& He, 2005; Williams, Liddell, et al., 2004) have found evidence for object-selective neural activity using strict criteria for lack of awareness, such as chance performance in discriminating 
the presence of objects from scrambled objects or blank displays. More commonly, studies have employed a variety of criteria consistent with a substantial degree of residual awareness, including relatively informal postexperiment questioning (Kranczioch et al., 2005; Marois, Do-Joon, \& Marvin, 2004; Pins \& Ffytche, 2003; Moutoussis \& Zeki, 2002) and forced-choice discrimination on high-level features, such as face identity (Kouider, Eger, Dolan, \& Henson, 2009). One especially common approach has been to use trial-by-trial forced-choice judgments of presence or absence and to focus analysis on trials where objects are judged to have been "absent" (Kouider et al., 2009; Henson, Mouchlianitis, Matthews, \& Kouider, 2008; Shapiro et al., 2007; Kranczioch et al., 2005; Marois et al., 2004; Pessoa \& Ungerleider, 2004; Beck et al., 2001). Typically, participants are not asked on each trial to report their confidence in judgments of presence or absence. The difficulty with this procedure concerns the unknown response criterion used as the basis for the "absent" judgment. If subjects perform with a conservative decision criterion, then objects that produce brief or weak impressions may lead to a decision of "absent," but, given some conscious impression, could still elicit object-specific brain activity.

To examine this possibility, we carried out an eventrelated fMRI and an ERP study using a common facemasking paradigm. By combining both methods, we aimed to maximize sensitivity, on the one hand profiting from the spatial resolution of fMRI and on the other using ERP to distinguish activity at different points in time. For example, brief brain activations that result in small metabolic demands could have measurable effects on ERPs but not on fMRI (Furey et al., 2006; Buxton, Wong, \& Frank, 1998). Faces were chosen as stimuli because of the known advantage of being processed in specific and well-studied anatomical areas (Haxby, Hoffman, \& Gobbini, 2000; Kanwisher, McDermott, \& Chun, 1997) and also of being associated with a well-characterized electrophysiological marker-the N170 (Bentin, Allison, Puce, Perez, \& McCarthy, 1996). On each trial, participants were asked to make a direct report on subjective awareness of a face using a 4-point scale. Interest was particularly focussed on trials where a face stimulus was presented, but participants reported having "no impression" of a face. We hypothesized that, when faces were truly nonperceived using this strict behavioral criterion, face-related brain activity would be weak or absent. Our results suggest close similarity of face-related activity in ventral stream and fronto-parietal regions. In both cases, face-related activity is strongly dependent on conscious perception.

\section{EXPERIMENT 1: fMRI}

\section{Methods}

\section{Participants}

Eighteen participants (10 men, ages $42-63$ years) gave written informed consent to participate in the experiment. All participants had normal or corrected-to-normal vision and no history of ophthalmological, neurological, or psychiatric disorders. The study was approved by Cambridge University Psychological Ethics Committee.

\section{Stimuli and Procedure}

The stimuli were 105 grayscale photographs of celebrities and the same number of texture images built by scrambling the face set into random squares of $10 \times 10$ pixels. All stimuli were $200 \times 200$ pixels in size and were reduced to $70 \%$ of their contrast to make detection more difficult. A stimulus mask was also made by taking a scrambled image that was not included in the stimulation material and increasing the contrast by $10 \%$.

The experiment consisted of three event-related runs. Each run lasted approximately $9 \mathrm{~min}$ and contained 280 trials in total. In each run, 35 different faces and 35 different scrambled images were each presented once at four different exposure durations $(33,50,67$, or $83 \mathrm{msec}$ ). A trial started with the presentation of a fixation cross for $200 \mathrm{msec}$, which was followed by a face or a scrambled stimulus at one of the exposure durations and then immediately masked. The total combined duration of the stimulus and mask was fixed at $200 \mathrm{msec}$ (Figure 1). From the offset of the mask, participants had up to 1770 msec to make their response, after which the fixation cross of the next trial appeared. Participants were instructed to rate their perception using a 4-point scale: sure a face was presented, fairly sure a face was presented, possibly saw a face, and no impression of a face. Responses were made by pressing buttons underneath the left middle finger (sure), left index finger

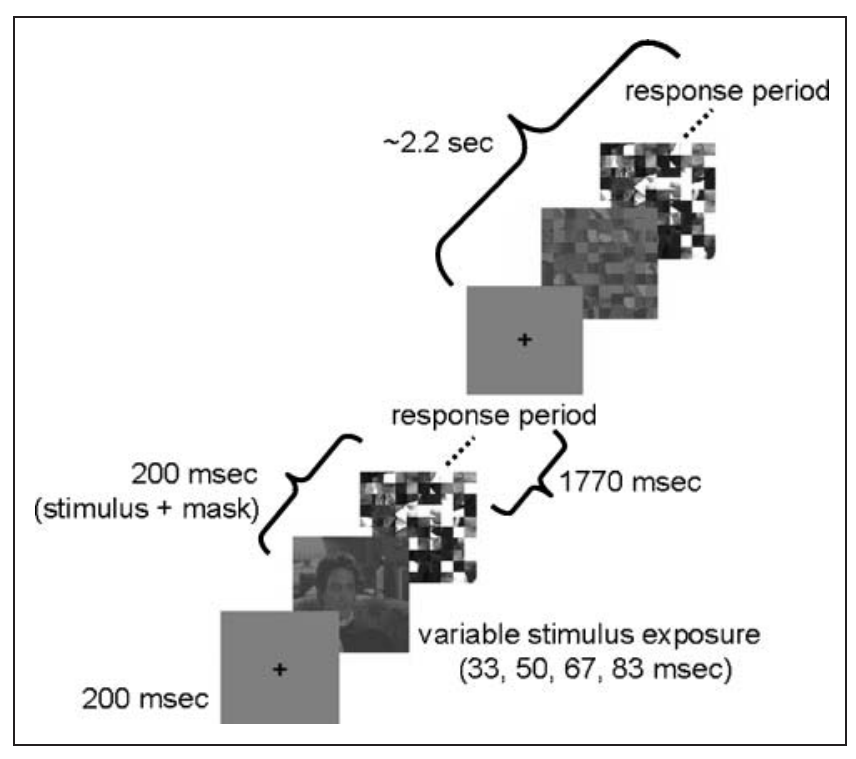

Figure 1. Experimental design. Each trial started with a black fixation cross (200 msec) followed by a face or a nonface stimulus (33-83 msec) that was immediately masked. Total stimulus + mask duration was fixed at $200 \mathrm{msec}$. The response could be made at any time during a $1770-\mathrm{msec}$ intertrial interval 
(fairly sure), right index finger (possibly), or right middle finger (no impression). The order in which different stimulus types (face or scrambled face) and stimulus durations were presented was randomized across runs. All images were presented at fixation using E-Prime (Psychology Software Tools, Inc., Pittsburgh, PA) and backprojected via an LCD projector onto a translucent screen located on the rear of the scanner. Participants viewed the stimuli using a mirror mounted on the head coil. Images subtended a visual angle of $3.26^{\circ}$.

Each event-related run was alternated with a run of a localizer task lasting $3.2 \mathrm{~min}$. The localizer runs contained two repetitions of three different stimulus blocks presented with intervening blocks of fixation on an empty screen. Stimulus blocks included one block of 10 faces (using a different set of faces from those employed in the event-related task), one block of 10 objects, and one block of 10 scrambled faces. Each block lasted for $16 \mathrm{sec}$, and participants were simply instructed to watch the stimuli without performing any task. Before starting the scanning session, all participants were instructed about the different task demands (event-related runs and localizer runs) and performed a short training session with a different set of images. The entire protocol including instruction and training lasted about an hour.

\section{fMRI Acquisition}

The scanning was performed on a 3T Tim Trio System (Siemens, Erlangen, Germany). Functional images were acquired using a gradient-echo EPI sequence (echo time $=30 \mathrm{msec}$, repetition time $=2 \mathrm{sec}$, field of view $=$ $192 \mathrm{~mm}$, flip angle $=78^{\circ}$ ). For each volume, we acquired 32 slices (matrix size $=64 \times 64$, voxel size $=3 \times 3 \times 3$ with $1-\mathrm{mm}$ interslice interval) with a pitch of $30^{\circ}$ up at the front to reduce susceptibility aartifacts in temporal cortices (Deichmann, Gottfried, Hutton, \& Turner, 2003). High-resolution structural images were obtained using a T1-weighted MPRAGE sequence with $1 \times 1 \times 1 \mathrm{~mm}$ spatial resolution (acquisition matrix $=256 \times 240 \times 160$ ), echo time $=2.99 \mathrm{msec}$, and repetition time $=2250 \mathrm{msec}$.

\section{Behavioral Analysis}

For analysis, trials were sorted into six awareness conditions, depending on participants' responses. Trials wherein a face was presented (face trials) were classified according to whether participants were sure or fairly sure that they had seen a face (Hit condition), were uncertain about face presentation but thought they had possibly seen a face (UF condition), or reported no impression of a face (Miss condition). Scrambled face trials were classified as false alarms if participants reported that they were sure or fairly sure of a face presentation (FA condition), uncertain trials if they responded they had possibly seen a face (US condition), and correct rejections if they reported no impression of a face (CR condition). One-way ANOVAs with the factor exposure (four exposure times) were carried out for each condition separately. The Huynh-Feldt correction was used to mitigate violations of the sphericity assumption in repeated measures (Jennings \& Wood, 1976), and the corresponding epsilon values $(\varepsilon h)$ are reported.

\section{MRI Analysis}

All functional images were processed using SPM5 software (Wellcome Department of Cognitive Neurology, London, UK). The first 14 volumes of each run were discarded to allow for T1 equilibration effects. Before statistical analysis, each participant's data were spatially realigned to the first volume, corrected for slice acquisition time delays, normalized to the Montreal Neurological Institute (MNI) template brain, and spatially smoothed using a Gaussian kernel (8 mm FWHM).

Statistical analysis of both the localizer task and the event-related runs was carried out using a general linear model (Friston, 1995; Worsley \& Friston, 1995). For the localizer task, data from each participant were analyzed using a separate fixed effects model to identify participantspecific fusiform face area (FFA) and occipital face area (OFA) ROIs. Each model contained three sessions, with each session corresponding to one of the sets of localizer runs. Within each session, each of the three block types was modeled by a separate regressor that was formed by convolving a timing function reflecting the onset and duration of those blocks with a canonical haemodynamic response function. Participant-specific realignment parameters were also included as covariates to account for movement-related variance.

A contrast between face blocks and scrambled face blocks was used to identify FFA and OFA ROIs for each participant. These were defined by taking all voxels within the left and right fusiform gyri and the left and right inferior occipital gyri that showed a significant difference at a threshold of $p<.001$ (uncorrected for multiple comparisons). Anatomical templates for the fusiform and inferior occipital gyri were obtained from the automated anatomical labeling (AAL) ROI library (Brett, Anton, Valabregue, \& Poline, 2002; Tzourio-Mazoyer et al., 2002) with the fusiform regions further truncated to restrict the search to the midfusiform gyrus $(x= \pm 55$ to $\pm 18, y=-70$ to $-30, z=-28$ to -5 ). Although it was possible to define the right FFA ROI in all participants, it was not possible to define the left FFA in two participants, the left OFA in five participants, and the right OFA in two participants (see Appendix A).

Additional ROIs covering fronto-parietal areas found to be active during many different tasks demanding cognitive control (Duncan, 2006; Miller \& Cohen, 2001; Duncan \& Owen, 2000) were defined as 10-mm-radius spheres centered on anatomical coordinates reported in previous studies (Dumontheil, Thompson, \& Duncan, 2011) using the MarsBaR toolbox (Brett et al., 2002; marsbar.sourceforge. 
net). These fronto-parietal ROIs comprised the ACC ( $x=0$, $y=31, z=24)$, pre-SMA $(x=0, y=18, z=50)$, inferior frontal sulcus (IFS; $x= \pm 41, y=23, z=29$ ), anterior insula/frontal operculum (AI/FO; $x= \pm 35, y=18, z=2$ ), and intraparietal sulcus (IPS: $x= \pm 37, y=-56, z=41$ ).

The first pass analysis of event-related data showed that spatial smoothing was causing cerebellar activation to fuse with fusiform cortex activation. To prevent this happening, we masked out the cerebellum from the normalized functional images for a second pass analysis. All the cerebellar and vermis AAL ROIs were combined using MarsBaR, and the remaining white matter structures in the cerebellum were filled in with MRIcro (Rorden \& Brett, 2000; www.mricro.com). The combination of the white matter fill and the AAL cerebellar ROIs was the whole of cerebellum mask (WoC). In addition, a mask (ToC) of cerebellum closest to the fusiform gyrus was also built by combining the "Cerebellum_Mid_L" and "Cerebellum_Mid_R" AAL areas. For every functional run, we calculated the mean of the voxel signal for all voxels within the ToC mask and all time points, giving a single run-specific ToC mean value. In each run, we replaced all voxel values in the WoC mask, with this ToC mean. In this way, we made the cerebellar signal invariant over the cerebellum and over time, but similar in absolute value to the fusiform activity. The aim of this procedure was to reduce the edge effect of smoothing across the fusiform/ cerebellar boundary. Finally, we smoothed the normalized images by $8 \mathrm{~mm}$ in the usual way.

Data from the event-related runs were then analyzed in two steps. First, a fixed effects model was constructed for each participant individually. Data from each of the three runs were included as a separate session, and each session was modeled using 28 regressors, one for each combination of response type, stimulus type, and exposure duration (as "sure" and "fairly sure" responses to scrambled faces were rare, these were combined into a single regressor, giving seven different response type by stimulus type conditions at each exposure). Regressors were formed by convolving timing functions indicating the onset of each stimulus type with a canonical haemodynamic response function. Once again, realignment parameters were also included as covariates to account for movement-related variance.

Both whole-brain random effect and ROI analyses were performed at the group level. Because response rates varied strongly with exposure duration (Figure 2), for individual participants, the full design of response type $\times$ exposure time contained many cells with few or no trialsin particular for the Miss response condition at medium and longer exposure times. For analyses, we therefore selected just two consecutive exposure times for each participant, chosen to minimize the occurrence of cells with small trial numbers. ${ }^{1}$ Selected times for each participant, called "best exposures," are specified in Appendix B, along with the numbers of trials for every combination of response type and exposure time.

For the random effects analysis, each of the three main contrasts of interest ("Hit vs. CR" [activation related to face perception], "UF vs. CR" [activation related to uncertainty when a face was presented], "Miss vs. CR" [activation related to no perception of a face when it was presented]) at the two individually selected exposures was submitted to a repeated measures ANOVA (rm-ANOVA) with factors event type (e.g., Hit vs. CR) and exposure duration. Data for FA and US response categories were not examined further. For whole-brain analysis, all reported activations survived correction for multiple comparisons (false discovery rate $[\mathrm{FDR}], p<.001$ ). For the ROI analysis, raw contrast values of each condition of interest (Hit, UF, Miss, CR) were computed in each defined region, followed by separate ANOVAs as above for each event type contrast and ROI.

A final analysis of FFA and OFA data addressed the possibility that unconscious processing may be more evident in the detailed intrasubject pattern of brain activity than in global activity levels (Sterzer et al., 2008). To this end, we used multivoxel pattern analysis (MVPA; see Stokes,
Figure 2. Behavioral results of Experiment 1. Response proportions for the different awareness conditions are represented as a function of stimulus duration. Errors bars indicate SEM.

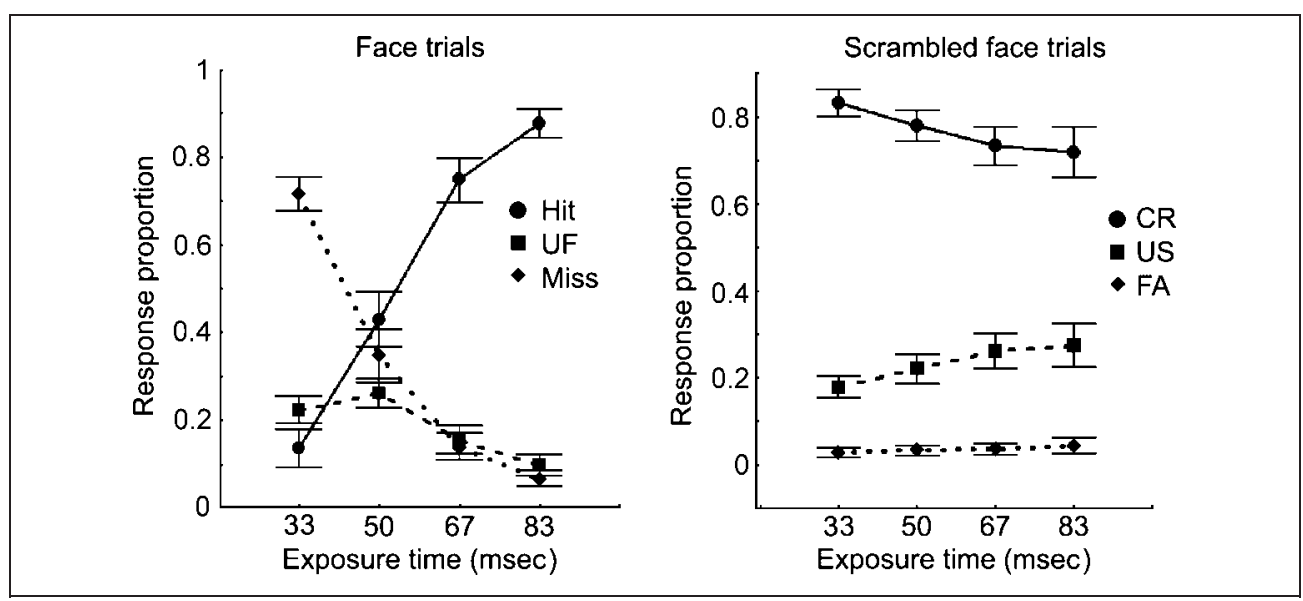


Thompson, Cusack, \& Duncan, 2009) to detect patterns of neural activity that could reliably differentiate between trials in which a face or scrambled image was presented. Initially, we verified that activity in FFA and OFA differentiates between face and scrambled stimuli when participants could accurately report the presence or absence of face stimuli (i.e., Hit vs. CR). For the critical test, however, we attempted to train our classifier to differentiate between scrambled stimuli and faces that were not seen (i.e., Miss vs. CR).

All pattern analyses were performed on minimally preprocessed images (realigned, slice-time corrected, but not spatially normalized or smoothed). Adapting a searchlight approach to MVPA (Kriegeskorte, Goebel, \& Bandettini, 2006), each analysis performed was centered on each voxel within the (individual-participant-defined) FFA to examine the pattern of activity measured within the surrounding cortical volume to include around 100 voxels using a 10-mm radius. Data were subdivided into separated train-test subsets according to a leave-one-runout cross-validation scheme, resulting in three train-test iterations. Classification was based on a correlation approach that additionally accounts for the multivariate structure of the error term (Kriegeskorte et al., 2006). First, we estimated the general linear model for the event-related task for each voxel within the analysis sphere separately for train and test data. We then performed condition-specific contrasts between beta estimates. For the aware analysis, the contrast between Hit and CR was calculated for two optimal exposure durations defined for each subject (see Results), and for the unaware analysis, the contrast was calculated between Miss and CR for the same two optimal exposure durations. Next, we scaled the resulting difference pattern according to the multivariate structure of the measurement noise by calculating the residual variance (i.e., observedfitted data) of the train data within the searchlight volume and then used a shrinkage estimator to estimate the covariance of the residual. We then divided the pattern of difference beta estimates by the square of the residual covariance matrix to define the conditiondiscriminative training pattern. We repeated these steps for the statistically independent test data and then evaluated the pattern match using a voxel-wise correlation between train and test patterns. A positive correlation between discriminative patterns was recorded as a correct classification, whereas zero or negative correlation coefficient was recorded as a misclassification. The procedure was repeated for all three cross-validation iterations, and the average classification score was recorded. For each participant, the full cross-validation cycle was repeated for searchlight spheres (radius $=10 \mathrm{~mm}$ ) centered on each voxel in the FFA/OFA, and average classification accuracy was calculated across these repetitions within each region. These final average accuracies were then entered into second-level group analysis (repeated measures $t$ test).

\section{Results}

\section{Behavioral Results}

Figure 2 shows the proportion of responses for each perceptual awareness condition as a function of stimulus duration. The proportion of correct face detections (Hits) significantly increased with exposure time $\left(F_{(3,51)}=96.71, p<.001, \varepsilon h=0.53\right)$, whereas the rate of missed faces was significantly reduced $\left(F_{(3,51)}=166.1\right.$, $p<.001, \varepsilon h=0.66)$. Uncertainty about face presentation (UF) was relatively low at all exposure times (less than $25 \%$ of responses) but significantly decreased with increasing exposure duration $\left(F_{(3,51)}=10.36, p<.002\right.$, $\varepsilon h=0.56)$.

Although the rate of CRs was high at all exposure times (Figure 2, right), it was significantly affected by stimulus visibility $\left(F_{(3,51)}=4.43, p<.01, \varepsilon h=0.47\right)$, showing some reduction with increasing exposure. The rate of US responses significantly increased over exposure time $\left(F_{(3,51)}=4.41, p<.01, \varepsilon h=0.48\right)$. The false alarm rate was low (less than $5 \%$ of scrambled face trials) and did not vary with exposure.

\section{Event-related fMRI Results}

Whole-brain analysis. The main goal of our study was to test whether the activation of ventral face responsive regions (FFA and OFA) and the selected fronto-parietal ROIs (see MRI Analysis under Materials and Methods) depended on conscious face perception. In particular, we asked whether those face trials wherein subjects reported no impression of a face elicited any significant face-selective activity. The whole-brain analysis showed a widespread activation during conscious face perception (contrast "Hit vs. CR," Table 1, Figure 3). In addition to an expected activation of right sensorimotor cortex (participants responded to perceived faces with their left hand), significant bilateral activations were seen in the lateral frontal cortex, the IPS, the insula, and the pre-SMA, as well as in left fusiform gyrus. Other significant activations included bilateral superior parietal lobule, middle temporal gyrus, and subcortical structures, including BG and thalamus. Uncertainty about face presentation ("UF minus CR") induced a pattern of activity that was broadly similar, although less widespread. Significant differences were observed bilaterally in the lateral frontal cortex, the insula, the IPS, the superior parietal lobule, the pre-SMA, and the left fusiform gyrus (Table 1).

In contrast to the above findings, faces that were not consciously perceived ("Miss minus CR") did not induce significant activations. When the significance threshold was reduced to $p<.01$ (uncorrected), only a cluster of voxels $(x=-36, y=2, z=-24)$ placed in a white matter region behind left superior temporal pole was activated.

ROI analyses. As can be seen in panels of Figure 3, activity was significantly higher for correctly detected faces 
Table 1. Experiment 1: Whole-brain Analysis

\begin{tabular}{|c|c|c|c|c|c|}
\hline \multirow[b]{2}{*}{ Region } & \multirow[b]{2}{*}{ Hemisphere } & \multicolumn{3}{|c|}{$\begin{array}{c}M N I \\
\text { Coordinates }\end{array}$} & \multirow[b]{2}{*}{$t$} \\
\hline & & $x$ & $y$ & $z$ & \\
\hline \multicolumn{6}{|l|}{ Hit vs. $C R$} \\
\hline \multirow[t]{2}{*}{ Superior frontal gyrus } & $\mathrm{L}$ & -20 & 12 & 54 & 3.92 \\
\hline & $\mathrm{R}$ & 26 & 12 & 52 & 4.02 \\
\hline \multirow[t]{3}{*}{ Middle frontal gyrus } & $\mathrm{L}$ & -40 & 44 & 0 & 4.15 \\
\hline & & -30 & 52 & 4 & 3.71 \\
\hline & $\mathrm{R}$ & 42 & 50 & 2 & 3.23 \\
\hline \multirow[t]{4}{*}{ Inferior frontal gyrus } & $\mathrm{L}$ & -48 & 26 & 28 & 4.72 \\
\hline & & -46 & 18 & 30 & 4.81 \\
\hline & $\mathrm{R}$ & 50 & 24 & 28 & 3.50 \\
\hline & & 56 & 16 & 30 & 3.51 \\
\hline \multirow[t]{2}{*}{ Inferior frontal/insula } & $\mathrm{L}$ & -28 & 24 & -2 & 4.09 \\
\hline & $\mathrm{R}$ & 32 & 22 & 0 & 4.84 \\
\hline \multirow[t]{2}{*}{ Pre-SMA } & $\mathrm{L}$ & -2 & 22 & 44 & 3.88 \\
\hline & $\mathrm{R}$ & 6 & 24 & 44 & 3.61 \\
\hline SMA & $\mathrm{R}$ & 10 & -14 & 52 & 4.46 \\
\hline Postcentral gyrus & $\mathrm{R}$ & 44 & -18 & 54 & 9.08 \\
\hline \multirow[t]{3}{*}{ Superior parietal lobule } & $\mathrm{L}$ & -8 & -68 & 48 & 4.28 \\
\hline & $\mathrm{R}$ & 22 & -56 & 68 & 3.75 \\
\hline & & 14 & -66 & 48 & 4.28 \\
\hline \multirow[t]{5}{*}{ Inferior parietal lobule } & $\mathrm{L}$ & -44 & -54 & 52 & 4.00 \\
\hline & & -60 & -48 & 30 & 3.03 \\
\hline & & -34 & -76 & 40 & 3.93 \\
\hline & $\mathrm{R}$ & 40 & -50 & 50 & 5.22 \\
\hline & & 38 & -72 & 38 & 5.27 \\
\hline Middle temporal gyrus & $\mathrm{L}$ & -60 & -46 & -8 & 2.62 \\
\hline Fusiform gyrus & $\mathrm{L}$ & -40 & -58 & -16 & 3.38 \\
\hline Putamen & $\mathrm{R}$ & 30 & -6 & -2 & 3.56 \\
\hline \multirow[t]{2}{*}{ Pallidum } & $\mathrm{L}$ & -14 & 10 & 0 & 2.75 \\
\hline & $\mathrm{R}$ & 14 & 10 & 0 & 3.93 \\
\hline Caudate & $\mathrm{L}$ & -12 & 6 & 8 & 2.88 \\
\hline Thalamus & $\mathrm{R}$ & 14 & -16 & 8 & 3.92 \\
\hline \multicolumn{6}{|l|}{$U F$ vs. $C R$} \\
\hline \multirow[t]{2}{*}{ Middle frontal gyrus } & $\mathrm{L}$ & -42 & 44 & 0 & 3.80 \\
\hline & $\mathrm{R}$ & 46 & 36 & 22 & 3.77 \\
\hline Inferior frontal gyrus & $\mathrm{L}$ & -50 & 18 & 30 & 4.21 \\
\hline \multirow[t]{2}{*}{ Inferior frontal/Insula } & $\mathrm{L}$ & -28 & 24 & 0 & 4.12 \\
\hline & $\mathrm{R}$ & 32 & 20 & 0 & 4.66 \\
\hline
\end{tabular}

Table 1. (continued)

\begin{tabular}{|c|c|c|c|c|c|}
\hline \multirow[b]{2}{*}{ Region } & \multirow[b]{2}{*}{ Hemisphere } & \multicolumn{3}{|c|}{$\begin{array}{c}M N I \\
\text { Coordinates }\end{array}$} & \multirow[b]{2}{*}{$t$} \\
\hline & & $x$ & $y$ & $z$ & \\
\hline Pre-SMA & $\mathrm{L}$ & -2 & 22 & 44 & 4.75 \\
\hline Precentral gyrus & $\mathrm{L}$ & -30 & 2 & 62 & 3.90 \\
\hline \multirow[t]{3}{*}{ Superior parietal lobule } & $\mathrm{L}$ & -12 & -70 & 50 & 4.52 \\
\hline & $\mathrm{R}$ & 34 & -58 & 46 & 4.40 \\
\hline & & 20 & -66 & 54 & 3.85 \\
\hline \multirow[t]{2}{*}{ Inferior parietal lobule } & $\mathrm{L}$ & -46 & -42 & 46 & 4.41 \\
\hline & $\mathrm{R}$ & 42 & -34 & 38 & 3.64 \\
\hline \multirow[t]{2}{*}{ Middle temporal gyrus } & $\mathrm{L}$ & -58 & -38 & 0 & 3.97 \\
\hline & & -56 & -46 & -10 & 3.50 \\
\hline Fusiform gyrus & $\mathrm{L}$ & -52 & -62 & -12 & 3.75 \\
\hline
\end{tabular}

Coordinates of activation peaks for regions showing significant increased activity (whole-brain correction $p<.001$ FDR) under condition of conscious face perception (contrast "Hit vs. CR") and uncertainty of face presentation (contrast "UF vs. CR"). All activations are reported in MNI coordinates.

$\mathrm{L}=$ left; $\mathrm{R}=$ right.

(Hits) than for correctly rejected nonfaces (CR) in the left FFA and the left OFA (Table 2). A similar trend in the right FFA was borderline ( $p=.1$, two tailed). In contrast, none of the ventral ROIs showed differential activation between UF trials and CR trials. The critical contrast of Miss and CR trials suggested a significant difference in the right OFA (Table 2). The direction, however, was opposite to expectation, with greater activity on CR (scrambled face) trials (Figure 3).

With the exception of ACC, all fronto-parietal ROIs showed increased activation for Hits and UFs compared with CRs (Figure 3; Table 2). No differences were found between activation in Miss and CR trials in any of the fronto-parietal ROIs.

There was generally little effect of exposure duration in these analyses, with significant main effects in just right FFA $\left(F_{(1,17)}=6.01, p<.03\right)$ for the Hit-CR analysis and bilateral IFS and AI/FO $(F>4.5, p<.05)$ for the MissCR analysis.

MVPA. Multivoxel pattern analyses performed on data from FFA and OFA were able to accurately classify whether a face or scrambled image was presented when participants could accurately report face presence or absence (Hits vs. CR: IFFA 79\% correct: $t(15)=9.8, p<.001$; rFFA 65\% correct: $t(17)=3.04, p=.01$; lOFA $72 \%$ correct: $t(12)=3.51$, $p=.004 ;$ rOFA $66 \%$ correct: $t(15)=2.8, p=.012)$. In contrast, activity patterns in these areas did not differentiate between the presentation of face or scrambled images on trials in which participants reported that they could 


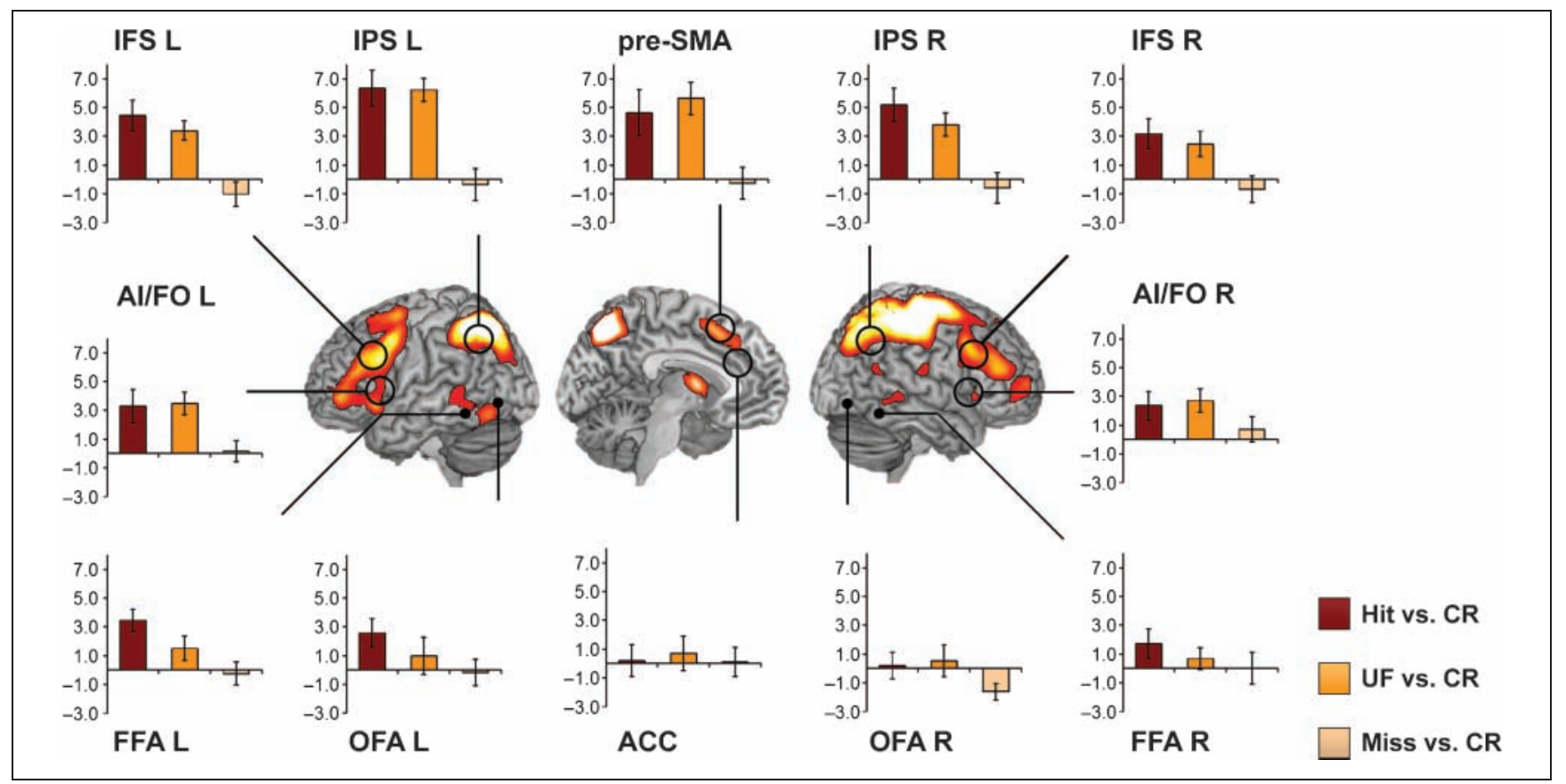

Figure 3. Group results of ROI and whole-brain analysis. Whole-brain render shows activation produced by the contrast "Hit versus CR" (at a whole-brain correction of $p<.001$ FDR). Panels show contrast values estimated for Hit, UF, and Miss conditions in the ROI analyses (L = left; $\mathrm{R}=$ right). Bars represent mean and standard error of the difference of each condition minus CR. Although certain or uncertain face perception (Hit or UF) induced a large activation in most ROIs, there was no differential activation for Miss versus CR.

not see any face, Miss and CR, respectively. Classification between Miss and CR trials was no better than chance (IFFA: $57 \%$ correct, $t(15)=1.12, n s ;$ rFFA: $48 \%$ correct, $t(17)=$ $-0.31, n s ;$ lOFA $55 \%$ correct: $t(12)=0.6, n s ;$ rOFA $41 \%$ correct: $t(15)=-1.59, n s)$ and was also significantly worse than classifier performance associated with the aware condition (seen $>$ unseen for IFFA: $t(15)=3.12$, $p=.01$; rFFA: $t(17)=3.22, p=.005$; lOFA: $t(12)=1.85$,

Table 2. Experiment 1: ROI Analysis

\begin{tabular}{|c|c|c|c|c|}
\hline \multirow[b]{2}{*}{$R O I$} & \multirow[b]{2}{*}{ Side } & \multicolumn{3}{|c|}{ Contrasts } \\
\hline & & Hit vs. $C R$ & $U F$ vs. $C R$ & Miss vs. $C R$ \\
\hline ACC & & $F_{(1,17)}=0.1, p=.8$ & $F_{(1,17)}=0.36, p=.6$ & $F_{(1,17)}=0.01, p=.9$ \\
\hline Pre-SMA & & $F_{(1,17)}=8.4, p=.01$ & $F_{(1,17)}=24.6, p=.0001$ & $F_{(1,17)}=0.05, p=.8$ \\
\hline \multirow[t]{2}{*}{ IFS } & $\mathrm{L}$ & $F_{(1,17)}=17.7, p=.001$ & $F_{(1,17)}=26.8, p<.001$ & $F_{(1,17)}=1.5, p=.2$ \\
\hline & $\mathrm{R}$ & $F_{(1,17)}=9.5, p<.01$ & $F_{(1,17)}=7.9, p=.01$ & $F_{(1,17)}=0.1, p=.5$ \\
\hline \multirow[t]{2}{*}{$\mathrm{AI} / \mathrm{FO}$} & $\mathrm{L}$ & $F_{(1,17)}=8.2, p=.01$ & $F_{(1,17)}=19.5, p<.0004$ & $F_{(1,17)}=0.04, p=.8$ \\
\hline & $\mathrm{R}$ & $F_{(1,17)}=5.7, p=.03$ & $F_{(1,17)}=10.9, p=.004$ & $F_{(1,17)}=0.6, p=.4$ \\
\hline \multirow[t]{2}{*}{ IPS } & $\mathrm{L}$ & $F_{(1,17)}=26.1, p=.0001$ & $F_{(1,17)}=56.7, p<.0001$ & $F_{(1,17)}=0.1, p=.8$ \\
\hline & $\mathrm{R}$ & $F_{(1,17)}=20.7, p<.0003$ & $F_{(1,17)}=22.9, p<.0002$ & $F_{(1,17)}=0.3, p=.6$ \\
\hline \multirow[t]{2}{*}{ FFA } & $\mathrm{L}$ & $F_{(1,15)}=20.4, p<.0004$ & $F_{(1,15)}=3.27, p=.1$ & $F_{(1,15)}=0.1, p=.8$ \\
\hline & $\mathrm{R}$ & $F_{(1,17)}=2.9, p=.1$ & $F_{(1,17)}=0.8, p=.4$ & $F_{(1,17)}=0.0002, p=1$ \\
\hline \multirow[t]{2}{*}{ OFA } & $\mathrm{L}$ & $F_{(1,12)}=6.8, p=.02$ & $F_{(1,12)}=0.6, p=.5$ & $F_{(1,12)}=0.03, p=.9$ \\
\hline & $\mathrm{R}$ & $F_{(1,15)}=0.05, p=.8$ & $F_{(1,15)}=0.2, p=.6$ & $F_{(1,15)}=6.94, p=.02$ \\
\hline
\end{tabular}

Main effects of event type for each contrast of interest and ROI.

$\mathrm{L}=$ left; $\mathrm{R}=$ right. 
$n s$; rOFA: $t(15)=3.19, p=.01)$. Once again, these results provide no evidence for unconscious face-selective activity on Miss trials.

\section{Discussion}

Results from ROI and whole-brain analyses were very consistent. Both kinds of analyses showed that conscious face perception (Hit) induced activation in ventral areas (FFA and OFA) as well as in a fronto-parietal network that involved lateral $\mathrm{pFC}$, IPS, $\mathrm{AI} / \mathrm{FO}$, and pre-SMA. In FFA and OFA, conventional univariate analysis revealed strong face-selective activity only in the left hemisphere, with a borderline effect in the right FFA. MVPA, however, confirmed the expected result of conscious face processing in both hemispheres.

More importantly, the results revealed that, when participants reported no impression of a face (Miss), brain activation did not differ from the pattern associated with a nonface presentation (CR). This was true both in conventional univariate activity analysis and in MVPA of individually determined FFA and OFA ROIs. The only exception was a suggestion of greater activity for CRs, that is, trials on which no face was presented, compared with Miss trials in the right OFA. Conceivably, this is a real result; for example, it could be that Miss trials were associated with some active inhibition of OFA activity. Given the unexpected direction of the difference, however, we are tempted to interpret it as a false positive; an interpretation strengthened by the lack of Miss-CR discrimination, in this region as in others, in the MVPA analysis. Otherwise, in our study there was no evidence of selective brain responses to faces that were not consciously perceived. In contrast, uncertainty (UF) induced significant brain activations similar to, but weaker than, those associated with Hits.

Absence of face-selective activity for Miss trials is important only if the experiment was sufficiently sensitive to detect such activity when it was present. This could be a particular concern as regards weak or absent univariate face-selective activity, seen in the contrast "Hit versus CR," in the right FFA and OFA. More broadly, however, the contrast of "Hit versus CR" shows several sensitive indices of face-selective activity in our data, in univariate activity for the left FFA/OFA, in MVPA analyses of both hemispheres, and in multiple regions of frontal and parietal cortex. On Miss trials, all of these were entirely absent.

In contrast to the results shown here, a number of previous studies on visual processing and awareness have found significant activation of ventral stream areas for trials on which faces or other stimuli were judged to be absent. Although activity in those studies has been generally higher on seen than on unseen trials, some activation has also been induced by unseen events. In a binocular color fusion experiment with house and face stimuli, Moutoussis and Zeki (2002) found that the same house-specific and face-specific areas were activated by seen and unseen stimuli, although for unseen stimuli activity was lower. Similar results were found by Dehaene et al. (2001) in a study using masked words and by Beck et al. (2001) in a study of change detection. Activation in relation to unconscious processing has also been observed in attentional blink paradigms. In these studies (Shapiro et al., 2007; Kranczioch et al., 2005; Marois et al., 2004), stronger parahippocampal activation was obtained on trials wherein participants did not detect a target house than on correct rejections of nonhouse events. The same result (Kranczioch et al., 2005) has also been reported for ACC.

Differential activations for missed events as compared with correct rejections suggest that unperceived stimuli are processed to some extent but do not reach the level of activity necessary to generate a conscious percept (Moutoussis \& Zeki, 2002). These studies have also proposed that the neural distinction between conscious and unconscious perceptions occurs at later stages of the information processing pathway, specifically in relation to fronto-parietal activation (Marois et al., 2004; Rees, Kreiman, \& Koch, 2002; Beck et al., 2001; Dehaene et al., 2001). However, it cannot be excluded that the persistence in these studies of some degree of activity in ventral stream areas during unawareness of event presentation could have been because of an assessment of participant stimulus visibility that underestimates conscious perception. In most of these studies (Kranczioch et al., 2005; Marois et al., 2004; Moutoussis \& Zeki, 2002; Dehaene et al., 2001), participants were not asked on each trial to report certainty of stimulus presence or absence. It is possible that under such conditions participants were induced to report as not seen stimuli of which they had some residual awareness (Hannula, Simous, \& Cohen, 2005). If that were true, then brain activity related to misses would have also included some unknown proportion of trials with some weak and, possibly, brief impression of stimulus presence (Wolfe, 1999). Our results in the UF condition support this idea. In the present study, trials wherein participants were not sure but considered that a face could have been presented yielded a strong significant activity in fronto-parietal and left FFA regions. Such activity could have been pooled together with Miss trials if we had employed a simple forced-choice present/ absent strategy to collect participants' responses.

A partially similar result to ours has been observed in a recent binocular rivalry study (Sterzer et al., 2008). In the present study, participants reported on each trial how confident they were about their percept. With this rigorous assessment of awareness, no differential activation was found in high-level visual areas for stimuli that were not consciously perceived. In contrast to our findings, however, the authors could distinguish between patterns of activity for invisible faces or houses when MVPA was employed, a finding that suggests that neural selectivity is preserved in the absence of differential brain activation (Sterzer et al., 2008). We return to this result in the General Discussion. 
In our study, the pattern of activation of the ventral stream and fronto-parietal regions was similar, both showing activation only for consciously detected faces. Although it is sometimes proposed that fronto-parietal activity is specifically involved in the generation of the conscious percept, this alone does not explain our results, because, on correct trials, there was certainly a conscious percept, either of a face or a scrambled face. It remains to be explained why fronto-parietal activity would be specifically driven by the detected face stimulus. A variety of previous studies show that fronto-parietal activity is selectively driven by target, unexpected, or otherwise special stimuli in a specific task context (Hampshire, Thompson, Duncan, \& Owen, 2008, 2009; Bledowski, Prvulovic, Goebel, Zanella, \& Linden, 2004; Downar, Crawley, Mikulis, \& Davis, 2002). In our study, subjects were set to look out for faces and, on many short-exposure trials, did not see them even when they were present. Detection of a target stimuli, rather than a general, nonspecific mechanism underlying conscious access, seems the most plausible explanation for face-related fronto-parietal activity.

In contrast to the other fronto-parietal areas included in our ROI analyses, ACC did not show differential activation when a face was perceived. Anterior cingulate has been described as part of a characteristic fronto-parietal network that is activated by a wide range of different cognitive challenges, including task switching, task novelty, episodic memory, complex response mapping, response conflict, working memory, and perceptual difficulty (Duncan, 2006; Ullsperger \& von Cramon, 2001; MacDonald, Cohen, Stenger, \& Carter, 2000; Carter et al., 1998). Despite the relative perceptual difficulty present in our task, it is possible that the absence of response competition or necessity to adjust task configuration and especially the absence of error processing could have reduced ACC involvement in our task.

\section{EXPERIMENT 2: ERP}

\section{Methods}

\section{Participants}

Eleven subjects ( 5 men, ages 43-60 years) participated in the study as volunteers. All subjects had normal or corrected-to-normal vision and no history of ophthalmological, neurological, or psychiatric disorders.

\section{Stimuli and Procedure}

Except for the following details, the stimuli and task design were identical to those described for Experiment 1. The stimulus set comprised 175 faces and 175 scrambled faces. All images were presented centrally using E-Prime (Psychology Software Tools, Inc., Pittsburgh, PA) on a CRT monitor and subtended a visual angle of $2.1^{\circ}$. The experiment took place in a room with dim illumination. Subjects were instructed to maintain fixation and minimize body movements and blinks during recording. The experiment was performed in one session, which comprised 1400 trials.

\section{EEG Recording}

Electrophysiological data were acquired using a 64-channel recording system (BrainAmp, BrainProducts $\mathrm{GmbH}$, Gilching, Germany). High-density ERPs were obtained using a cap for electrode positioning (Easycap $\mathrm{GmbH}$, Hersching, Germany) according to the extended 1020 system. The position of the cap on each subject's head was checked to ensure correspondence of marker electrodes with skull landmarks. All active electrodes (60 electrodes on the scalp) were referenced to a cephalic electrode placed on the vertex. Interelectrode impedance was always kept below $15 \mathrm{~kW}$. EOG was recorded with four electrodes placed on the outer canthus of each eye and $1 \mathrm{~cm}$ above and below the left eye. The EEG signal was filtered between 0.2 and $250 \mathrm{~Hz}$ and digitized with a sample rate of $500 \mathrm{~Hz}$ for off-line analysis. In each trial, marks corresponding to stimulus events and the subject's responses were coregistered with the EEG.

\section{Behavioral Analysis}

The analysis was the same as described for Experiment 1.

\section{ERP Analysis}

ERP data were analyzed only for a single stimulus exposure duration $(50 \mathrm{msec})$. After sorting the trials according to behavior and removing artifacts, this was the only exposure duration where the ERPs for each condition of interest (Hit, Miss, and CR) in each subject were based on a sufficient number of events (a minimum of 20 trials). UF was not included in the analysis because of insufficient data in most participants.

The continuous EEG record was segmented with a $100-$ msec prestimulus baseline and 600-msec poststimulus epoch. As in the behavioral analysis, trials were sorted by stimulus (face vs. scrambled) and response, once again combining response categories 1 and 2 (sure and fairly sure). Each EEG segment was then inspected both semiautomatically and visually; trials with artifacts (ocular movements, blinks) were rejected, and bad channels were linearly interpolated. For each subject, averaged ERPs time-locked to the stimulus presentation were obtained for each of the different stimulus-response conditions at each of the recording sites. These subject average waveforms were low-pass filtered $(30 \mathrm{~Hz})$ and baseline-corrected by subtracting the prestimulus amplitude value. Grandaveraged ERPs were calculated across subjects for each site and condition. Average waveforms were rereferenced to a common average reference.

Initial inspection suggested that three components of the ERPs, two negativities at occipito-temporal electrodes and a positivity at central electrodes, showed a differential 
response to face and scrambled face presentations. These components were identified as the N170 (N1), a second negativity (N2) peaking after $300 \mathrm{msec}$, and a slow positivity (SP) after $400 \mathrm{msec}$ (Figure 5A) and were chosen for subsequent analysis. The amplitude of these components was measured as the mean voltage determined within a 30-msec time window centered on the peak of the component for each condition and for each subject.

Twelve electrode clusters were defined (C1 = [FC1FC3-F1-F3], C2 = [FC2-FC4-F2-F4], C3 = [AF3-AF7-F5], $\mathrm{C} 4=[\mathrm{AF} 4-\mathrm{AF} 8-\mathrm{F} 6], \mathrm{C} 5=[\mathrm{C} 1-\mathrm{C} 3-\mathrm{CP} 1], \mathrm{C} 6=[\mathrm{C} 2-\mathrm{C} 4-$ $\mathrm{CP} 2], \mathrm{C} 7=[\mathrm{P} 5-\mathrm{P} 7-\mathrm{TP} 7], \mathrm{C} 8=[\mathrm{P} 6-\mathrm{P} 8-\mathrm{TP} 8], \mathrm{C} 9=[\mathrm{PO} 3-$ $\mathrm{PO} 7-\mathrm{O} 1], \mathrm{C} 10=[\mathrm{PO} 4-\mathrm{PO} 8-\mathrm{O} 2], \mathrm{C} 11=[\mathrm{AFz}-\mathrm{Fz}], \mathrm{C} 12=$ [Cz-CPz]), in which N1, N2, and SP amplitudes were averaged across electrodes for each condition. The resulting amplitude values of each component were submitted to rm-ANOVAs based on different planned contrasts of awareness and region (electrode clusters). Two contrasts were tested: Hit versus CR (amplitude modulation related to face perception) and Miss versus CR (amplitude modulation related to no perception of a face when it was presented). Analyses were conducted separately for the anterocentral (C1-C4, C11), central (C5, C6, C12), and posterior (C7-C10) clusters. An additional analysis of N1, N2, and SP scalp topography was carried out using the complete recording montage. With that purpose, a paired $t$ test was performed comparing the amplitude of each component in the two interest conditions (Hit vs. CR, Miss vs. CR). Statistical parametric maps of the components' scalp distribution were constructed. These maps were then thresholded using a criterion of $q<$ 0.05 FDR.

\section{Source Modeling}

A Bayesian model averaging approach (BMA; TrujilloBarreto, Aubert-Vazquez, \& Valdes-Sosa, 2004) was used to estimate the underlying generators of N1, N2, and SP. In this approach, multiple competitive primary current density (PCD) images are obtained by confining the putative EEG generators to different anatomical compartments. These are derived by coregistration of the MNI probabilistic brain atlas and the realistic digital brain phantom (Collins et al., 1998). Each anatomical constraint is regarded as a given model for source reconstruction. A model-independent image of the underlying brain electrical activity was then obtained by averaging all computed PCDs weighted by the corresponding support that they received from the data. This weighting coefficient is measured in terms of the posterior probability of the corresponding model (anatomical constraint), given the data.

The PCD was estimated for each ERP component after averaging across participants activity in the same time windows used in the ERP analysis. The recording montage was referenced to the cephalic electrode on the vertex. Additional anatomical constraints were imposed by restricting allowable source configurations to lie on and to be perpendicular to the cortical mantle. The source space was defined as a mesh of 5656 nodes (representing possible generators or sources) corresponding to the vertices of the triangles obtained by tessellation of the gray/white matter interface of the realistic digital brain phantom developed at the MNI (Collins et al., 1998). The forward model calculations were based on the three-shell spherical head model (Rush \& Driscoll, 1969). The center and radius of the spheres were fitted to the scalp, skull, and cerebral tissue of the same brain. The electrode coordinates were derived by coregistration of the electrodes' system and the digital brain phantom used and then projection onto the outermost sphere.

The PCD obtained for the CR condition was subtracted from the PCD obtained for the Hit condition, and the PCD difference was then normalized to the maximum positive and negative activities. Results are shown in brain renders where only sources that survived a threshold of $30 \%$ map maximum are displayed. Coordinates of the center of gravity of the activated clusters are reported in MNI space.

\section{Results}

\section{Behavioral Results}

The proportion of responses for each perceptual awareness condition as a function of stimulus duration is shown in Figure 4. Performance was very similar to that described for Experiment 1. Face detection significantly increased with exposure time $\left(F_{(3,30)}=75.5, p<.001, \varepsilon h=0.59\right)$, whereas the rate of missed faces was significantly reduced $\left(F_{(3,30)}=46.4, p<.001, \varepsilon h=0.41\right)$. Uncertainty about face presentation (UF) was low at all exposure times, although once again showed a significant decrease with increasing exposure $\left(F_{(3,30)}=9.03, p<.001, \varepsilon h=0.69\right)$. Correct rejection of scrambled faces was generally high at all exposure times (Figure 4, right) but decreased significantly with increasing exposure $\left(F_{(3,30)}=10.1, p<.001\right.$,, $b=$ 0.98). This reduction in accuracy was accompanied by a significant increase in both US and FA responses (US: $F_{(3,30)}=7.35, p<.002, \varepsilon h=0.66$; FA: $F_{(3,30)}=5.37$, $p<.004, \varepsilon h=0.73)$.

\section{ERP Results}

Correct detection of face presentation evoked three main components. Two of these showed maximum amplitude over posterolateral regions, the expected N170 (named here as N1) and N2 with a maximum amplitude around 320 msec (Figure 5A, left). A further slow positive component with a peak amplitude around 415 msec was observed over left central regions (SP; Figure 5A, right). The plots in Figure $5 \mathrm{~B}$ represent an interpolation of the scalp voltage distribution obtained by subtracting CR from Hit waveforms (left) and the corresponding statistic map (right) showing the results of a two-tailed $t$ test against 0 
Figure 4. Behavioral results of Experiment 2. Response proportions for the different awareness conditions are represented as a function of stimulus duration. Errors bars indicate $S E M$.

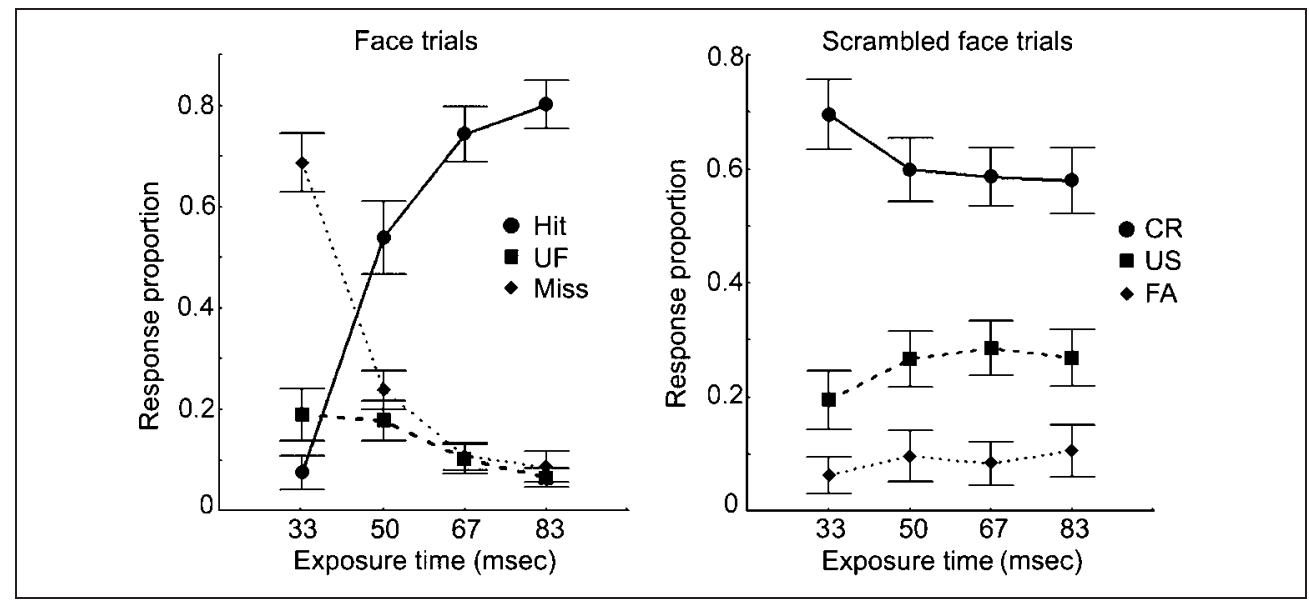

(thresholded at $q(\mathrm{FDR})<0.05)$. There was a rather symmetrical distribution for N1 on occipito-temporal electrodes (Figure 5B, top left) and a moderate lateralization to the right for $\mathrm{N} 2$ on occipitoparietal electrodes (Figure $5 \mathrm{~B}$, left middle). Also significant were anterior and central positivities, occurring at the same time as N1 and N2 and mirroring their spatial distribution. Central positivity, especially that coinciding in latency with N1, was interpreted as a vertex positivity, a positive component previously reported in the N170 literature (Jeffreys, 1989, 1996), that resembles the posterior negativity in both latency and response to experimental manipulations (Joyce \& Rossion, 2005). For the purpose of this study, the two components were considered together as a single com- ponent that reverses polarity from posterior to anterior regions. The SP distribution showed a lateralization to the left frontocentral electrodes (Figure 5B, bottom left) with a significant maximum on C3 (Figure 5B, bottom right).

Grand-averaged ERPs obtained on Hit, Miss, and CR trials are shown in Figure 6. The rm-ANOVA (Table 3) showed that N1 amplitude was significantly higher on Hit than on CR trials in all clusters and that there was no difference in N1 amplitude between Miss and CR trials. Similar results (Table 3 ) were obtained in the rm-ANOVA on N2 and SP amplitude. N2 amplitude was significantly higher on Hit than on CR trials, but there was no difference in N2 amplitude between Miss and CR trials. SP amplitude was significantly higher for Hits than CRs on left and central
Figure 5. Modulation of facerelated electrophysiological components with conscious perception. (A) Grand-averaged waveforms from electrodes P8 (left) and C3 (right). Compared with CRs, correct face detection (Hit) was associated with a larger N1 (N170) and also with the appearance of a late negativity, N2, around $300 \mathrm{msec}$, followed by an SP around $415 \mathrm{msec}$. Voltage scalp maps (B, left) and $t$ statistic topography - thresholded at $q$ (FDR) $<0.05-$ (B, right $)$ showed that both negativities had a posterior topographic distribution on occipitotemporal electrodes while the positivity showed a left lateralization on central derivations.

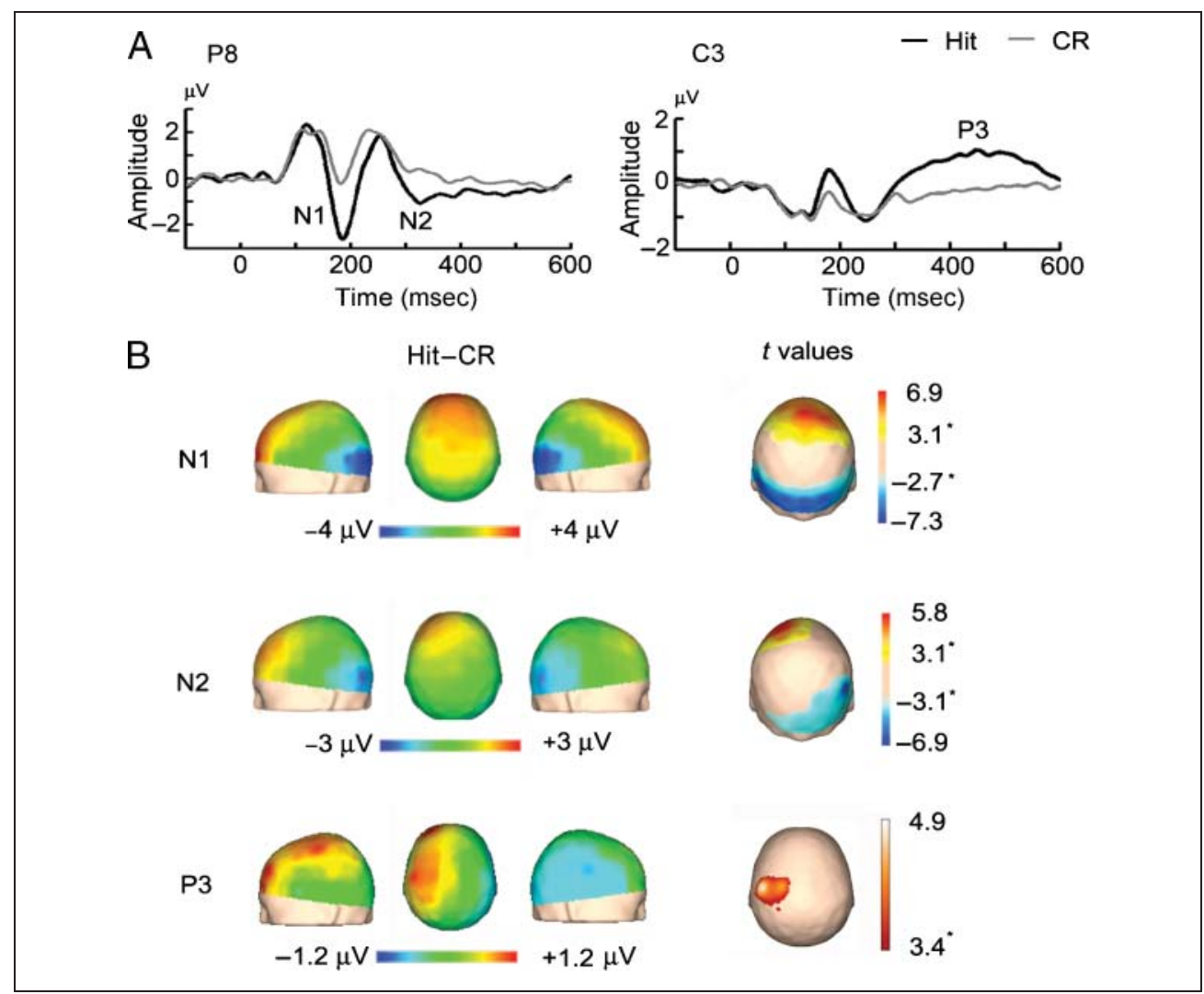




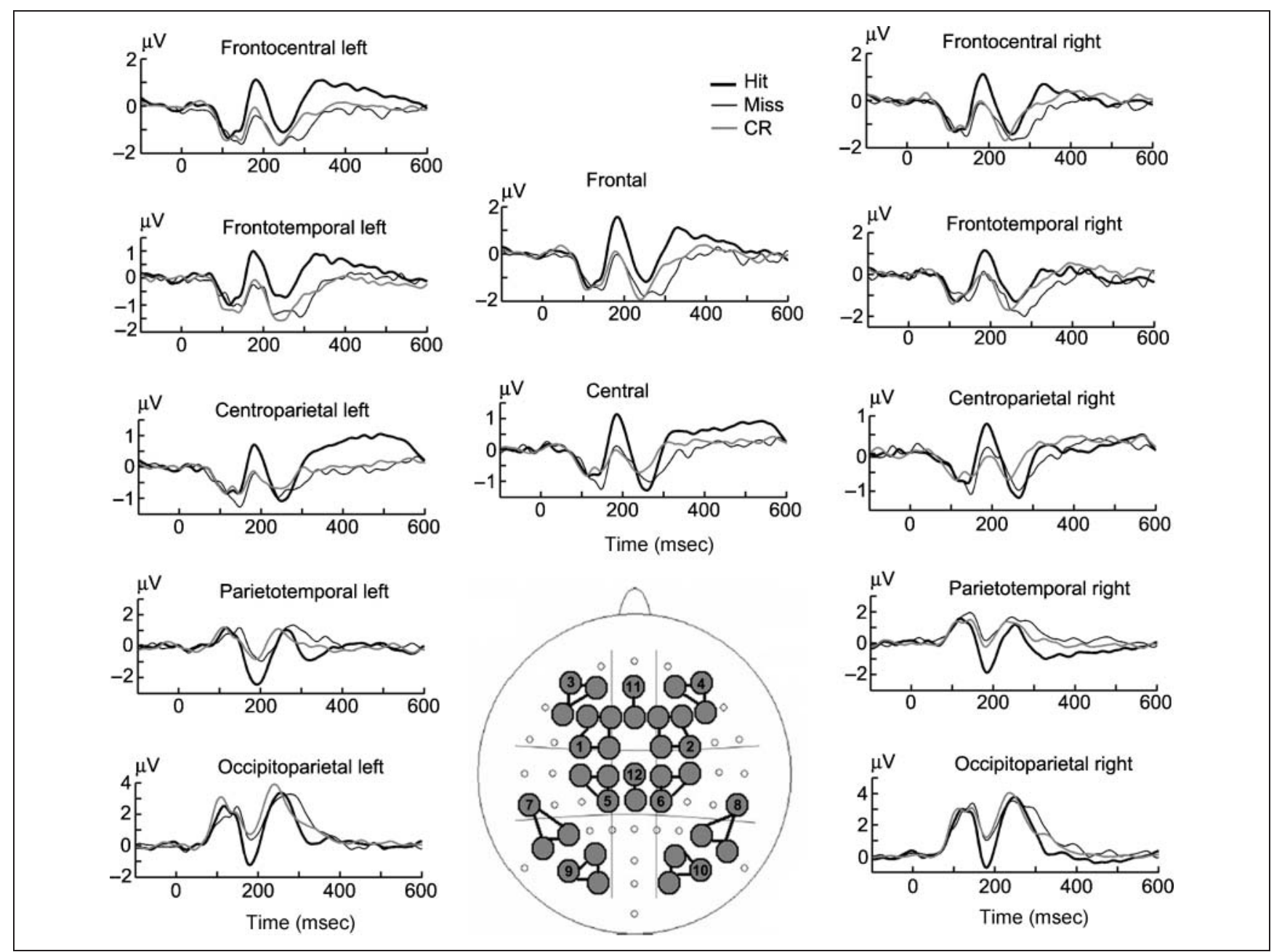

Figure 6. Grand-averaged ERPs obtained on Hit, Miss, and CR trials are shown for each electrode cluster included in the statistical analysis. Hits were associated with a larger N1 and N2 than Miss and CR in all clusters. SP was also larger for Hit compared with Miss and CR in left anterocentral and central clusters. Cluster definition is shown at the bottom center: $\mathrm{C} 1 / \mathrm{C} 2=$ left/right frontocentral; $\mathrm{C} 3 / \mathrm{C} 4=$ left/right frontotemporal; $\mathrm{C} 5 / \mathrm{C} 6=$ left/right centroparietal; $\mathrm{C} 7 / \mathrm{C} 8=$ parietotemporal; $\mathrm{C} 9 / \mathrm{C} 10=$ left/right occipitoparietal; $\mathrm{C} 11=$ midline frontal; $\mathrm{C} 12=$ midline central.

Table 3. Experiment 2: ERP Analysis

\begin{tabular}{|c|c|c|c|}
\hline \multirow[b]{2}{*}{ ERP Component } & \multirow[b]{2}{*}{ Cluster } & \multicolumn{2}{|c|}{ Contrasts } \\
\hline & & $H i t-C R$ & Miss-CR \\
\hline \multirow[t]{3}{*}{ N1 } & $1-4,11$ & $F_{(1,10)}=45.6, p<.0001$ & $F_{(1,10)}=1.2, p=.3$ \\
\hline & $5,6,12$ & $F_{(1,10)}=43.9, p<.0001$ & $F_{(1,10)}=0.4, p=.5$ \\
\hline & $7-10$ & $F_{(1,10)}=49.3, p<.0001$ & $F_{(1,10)}=0.8, p=.4$ \\
\hline \multirow[t]{3}{*}{ N2 } & $1-4,11$ & $F_{(1,10)}=22.4, p=.001$ & $F_{(1,10)}=1.8, p=.2$ \\
\hline & $5,6,12$ & $F_{(1,10)}=15.9, p=.003$ & $F_{(1,10)}=0.7, p=.4$ \\
\hline & $7-10$ & $F_{(1,10)}=20.6, p=.001$ & $F_{(1,10)}=0.5, p=.5$ \\
\hline \multirow[t]{5}{*}{ SP } & $1,3,11$ & $F_{(1,10)}=10.8, p=.01$ & $F_{(1,10)}=0.2, p=.7$ \\
\hline & 2,4 & $F_{(1,10)}=0.3, p=.6$ & $F_{(1,10)}=1.1, p=.3$ \\
\hline & 5,12 & $F_{(1,10)}=10.7, p=.01$ & $F_{(1,10)}=0.3, p=.6$ \\
\hline & 6 & $F_{(1,10)}=0.1, p=.8$ & $F_{(1,10)}=0.04, p=.8$ \\
\hline & $7-10$ & $F_{(1,10)}=1.2, p=.3$ & $F_{(1,10)}=1.5, p=.2$ \\
\hline
\end{tabular}

rm-ANOVA results for N1, N2, and SP components. 


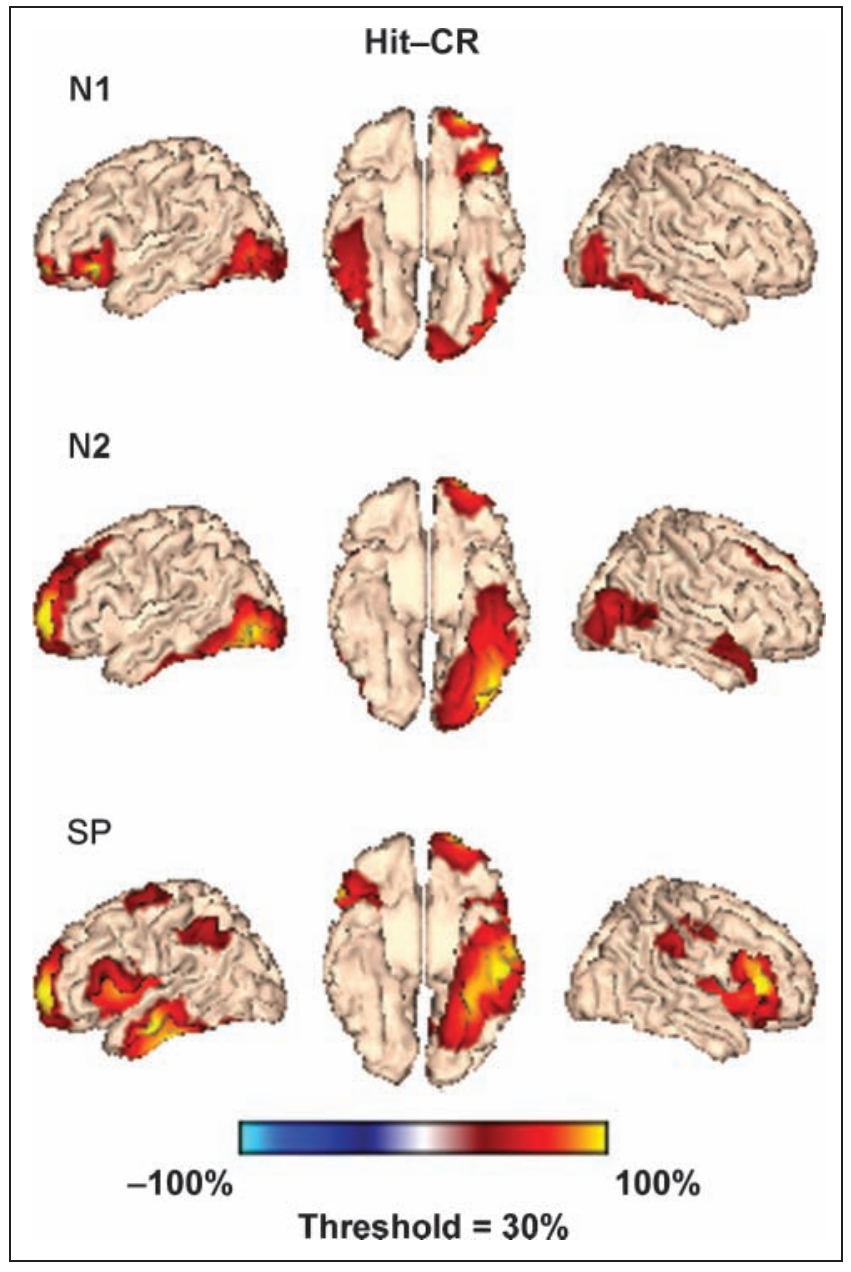

Figure 7. Brain renders of the contrast Hit minus CR PCDs at the time windows of N1, N2, and SP. The inverse solution was normalized to the maximum positive and negative activities, and then only sources that survived a $30 \%$ threshold were displayed.

clusters (Clusters 1,3,5,11,and 12), but as for N1 and N2 components, no differences were found between SP amplitude on Miss and CR trials.

\section{Source Analysis}

Given that only the conscious perception of faces (Hit vs. $\mathrm{CR}$ ) produced significant modulation of $\mathrm{N} 1, \mathrm{~N} 2$, and $\mathrm{SP}$ amplitude, source analysis was only carried out for these two conditions. The PCD obtained for CR trials was subtracted from the PCD obtained for Hits in each temporal window. The active sources that survived a 30\% thresholding are shown on brain renders in Figure 7. Center of mass coordinates are summarized in Table 4.

At the time of N1 occurrence, the main sources were estimated to lie in anterior and posterior ventral areas, including the right fusiform and inferior temporal gyri, bilateral middle and inferior occipital gyri, as well as left OFC and insula. Later, at the time of N2, activations were strongest in the left hemisphere included the lingual, inferior occipital, and fusiform gyri, as well as the inferior
Table 4. Experiment 2: Source Analysis

\begin{tabular}{lcrrr}
\hline & & \multicolumn{2}{c}{ MNI Coordinates } \\
\cline { 3 - 5 } Region & Hemisphere & \multicolumn{1}{c}{$x$} & $y$ & $z$ \\
\hline$N 1$ & & & & \\
Superior OFC & $\mathrm{L}$ & -25 & 59 & -8 \\
Inferior OFC & $\mathrm{L}$ & -45 & 29 & -4 \\
Insula & $\mathrm{L}$ & -28 & 19 & 6 \\
Fusiform & $\mathrm{R}$ & 43 & -47 & -15 \\
Inferior temporal & $\mathrm{R}$ & 49 & -28 & -23 \\
Middle occipital & $\mathrm{L}$ & -49 & -79 & 10 \\
& $\mathrm{R}$ & 42 & -83 & 15 \\
Inferior occipital & $\mathrm{L}$ & -45 & -73 & -4 \\
& $\mathrm{R}$ & 40 & -84 & -7 \\
Calcarine & $\mathrm{L}$ & -9 & -90 & -11
\end{tabular}

N2

$\begin{array}{llrrr}\text { Superior frontal } & \mathrm{L} & -25 & 61 & 5 \\ \text { Middle frontal } & \mathrm{L} & -32 & 31 & 45 \\ & \mathrm{R} & 35 & 26 & 40 \\ \text { Superior temporal pole } & \mathrm{R} & 49 & 6 & -19 \\ \text { Middle temporal } & \mathrm{R} & 51 & -63 & 11 \\ \text { Inferior temporal } & \mathrm{L} & -47 & -15 & -34 \\ \text { Fusiform } & \mathrm{L} & -36 & -41 & -20 \\ \text { Middle occipital } & \mathrm{R} & 43 & -78 & 15 \\ \text { Inferior occipital } & \mathrm{L} & -31 & -80 & -5 \\ \text { Lingual } & \mathrm{L} & -22 & -68 & -9\end{array}$

$S P$

Superior frontal

$-22 \quad 66 \quad 4$

Middle frontal

Precentral

Inferior frontal

Insula

Inferior parietal

Supramarginal

Middle temporal

Inferior temporal

Fusiform

Lingual

$-28-9$

$-36 \quad 56$

Coordinates of main activations obtained with BMA for regions showing increased activity during correct face perception (Hit vs. CR) in the time windows of N1, N2, and SP. All activations are reported in MNI coordinates.

$\mathrm{L}=$ left; $\mathrm{R}=$ right. 
and middle temporal gyri and, more dorsally, the superior and middle frontal gyri. In the right hemisphere, sources were located at the middle occipital and middle temporal gyri, extending anteriorly to the superior temporal pole and middle frontal gyrus. Finally, during the SP window, active sources persisted in the left ventral visual regions, but additional activations were also visible in the left middle temporal and inferior parietal gyri, as well as several regions of the frontal lobe and insula bilaterally (Table 4).

\section{Discussion}

Our results showed that perceptual awareness of face presentation was not only associated with a modulation of the N1 (N170) but also with the presence of an N2 around $320 \mathrm{msec}$ and an SP around $415 \mathrm{msec}$. There were some interesting similarities between N1 and N2. The amplitude of both components was higher for consciously perceived faces than for misses or scrambled face stimuli. The voltage distribution was also similar, with maximum negativity at posterior electrodes, and a complementary positivity at anterior electrodes. These similarities might suggest that the same neural generators are contributing to the origin of both negativities but are activated in different phases of conscious access to the face representation. Crucially, both components seemed uniquely associated with conscious face perception. Like the fMRI data, ERPs suggested no difference between Miss and CR trials, that is, no face-selective activity for nonconscious face presentations.

The occurrence of separate N1 and N2 components supports theories that propose a two-stage access to information for object recognition. It has been proposed that, at a first stage, objects are detected and categorized at their basic level (e.g., as face, house, dog, musical instrument), whereas at a second stage, recognition is at the subordinate level occurs (Grill-Spector \& Kanwisher, 2005). Evidence from object recognition studies in monkeys (Matsumoto, Okada, Sugase, Yamane, \& Kawano, 2005; Sugase, Yamane, Ueno, \& Kawano, 1999) suggests that object processing indeed occurs in two stages. Whereas early activity in inferotemporal neurons distinguishes face versus nonface stimuli, later activity distinguishes specific facial expressions or identities (Matsumoto et al., 2005; Sugase et al., 1999). Although recognition of face identity was not requested in our task, it is likely that, at least on many trials, participants did in fact recognise the famous faces they were shown. If that were the case, N1 could reflect perceptual evidence of face presentation in ventral face-specific areas during a first processing step_-previously termed as structural encoding (Zion-Golumbic \& Bentin, 2007; Bentin \& Deouell, 2000) — whereas N2 could signal access to a specific face representation in a second step (Bentin \& Deouell, 2000). A similar idea has been recently proposed by Nasr and Esteky (2009). On the other hand, the latency of SP and the fact that its amplitude was significantly higher for consciously detected faces - the target stimulus in the task-suggests a P300-like component, despite the left frontocentral distribution. It is likely that this component reflects postperceptual processes probably related to working memory and conscious access (Polich, 2007; Sergent, Baillet, \& Dehaene, 2005; Vogel, Luck, \& Shapiro, 1998).

Previous studies on object processing have reported similar modulations of early ERP components in relation to subjects' behavior. Liu, Harris, and Kanwisher (2002) found a difference in M170 (the magnetoencephalographic analogue of N170) for correct versus incorrect responses during a face recognition task. Another occipitotemporal negativity around 200-300 msec, named the visual awareness negativity, that shows a higher amplitude for hit than for miss trials has been described in different tasks involving object or letter categorization as well as line orientation judgments and simple stimulus detection (Koivisto et al., 2008).

Modulation of later negative components has also been found in several studies exploring the neural basis of face perception and recognition. Besides N170, another negativity with an occipito-temporal distribution and sensitivity to face familiarity, repetition, and priming (N250) has been described (Tanaka, Curran, Porterfield, \& Collins, 2006; Schweinberger, Pickering, Jentzsch, Burton, \& Kaufmann, 2002; Bentin \& Deouell, 2000). On the other hand, Bentin and Deouell (2000) described a negative component around $400 \mathrm{msec}$ that was modulated by face familiarity and showed a higher amplitude at frontocentral regions. According to these authors, the face-N400 familiarity effect could be associated with semantic activity involved in the identification of familiar faces. Similar familiar-facerelated components but with different scalp distributions have been described in other studies (Olivares \& Iglesias, 2008; Caharel, Fiori, Bernard, Lalonde, \& Rebai, 2006; Eimer, 2000). Finally, late positive components have also been observed in different tasks in relation to stimulus processing including face detection and identification (Lamy, Salti, \& Bar-Haim, 2009; Henson et al., 2003; Vogel et al., 1998).

Contrary to the findings described here, there are a group of studies (Jiang et al., 2009; Lamy et al., 2009; Sterzer et al., 2009; Henson et al., 2008; Williams, Liddell, et al., 2004) that have recently reported modulation of early and late electric or electromagnetic components in relation to events that were not consciously perceived. It cannot be excluded, however, that the unconscious related modulations observed in some of them (Lamy et al., 2009; Henson et al., 2008) originated from an awareness criterion that leaves open the possibility of some remaining conscious stimulus registration. Again, unreported fleeting perceptions cannot be ruled out with certainty if subjects are not allowed to report doubts on their perception in each trial but are instead forced to choose between two possible answers - seen or not, conscious or guessed (Lamy et al., 2009). Similarly, forced-choice tests of face identification may be at chance with much remaining 
awareness of face presence. We will return in the General Discussion to results (e.g., Jiang et al., 2009; Sterzer et al., 2009; Williams, Liddell, et al., 2004) that are not easily explained in this way.

It is widely known that the inverse solution problem of finding neural generators for a given scalp voltage distribution is both ill-posed-there is no unique solution for a given voltage configuration-and ill-conditioned-very sensitive to noise. Taken with caution, however, inverse solution methods allow tentative inferences on the generators of ERP components. In this study, significant sources contributing to the N1 during Hit trials were found in ventral visual areas, including mainly the right fusiform and bilateral inferior occipital gyri. Other generators were also identified in the left insula and OFC. There is no consensus in the literature on the underlying generators of the N170. Different studies of face processing have reported sources located in fusiform gyri, lateral occipital cortex (Deffke et al., 2007; Henson et al., 2003; Rossion, Joyce, Cottrell, \& Tarr, 2003), as well as in STS (Itier \& Taylor, 2004). It is possible that different generators contribute to the N170 component, depending on the task demands. Significant sources found in our study are consistent with generators described by a variety of previous studies (Sadeh, Podlipsky, Zhdanov, \& Yovel, 2010; Herrmann, Ehlis, Muehlberger, \& Fallgatter, 2005; Itier \& Taylor, 2004; Mnatsakanian \& Tarkka, 2004; Rossion et al., 2003; Bentin et al., 1996). Moreover, our results are also consistent with brain activations found in different functional brain imaging and intracortical recording studies of face perception. fMRI studies show robust activation during face perception in lateral fusiform gyrus, inferior occipital gyrus, superior temporal cortex, and OFC (Ishai, Schmidt, \& Boesiger, 2005; Hoffman \& Haxby, 2000; Halgren et al., 1999; Kanwisher et al., 1997). Recordings in patients with implanted electrodes have revealed facerelated activities in posterior and middle fusiform gyri as well as in middle temporal, inferior temporal, and inferior frontal gyri (Barbeau et al., 2008; Allison, Puce, Spencer, \& McCarthy, 1999). Agreement between our results and those described in the literature supports the plausibility of N1 generators found in the present study. Significant sources for the N2 were located in the inferior occipital, fusiform and middle temporal gyri, as well as superior temporal pole and frontal regions. Once again, these data support the suggestion that this negativity could be generated, in part, by the same neural sources as N1, but at a different stage of processing. Furthermore, modeled generators for SP in left inferior temporal cortex, middle frontal cortex, inferior parietal cortex, and bilateral inferior frontal cortex are in correspondence with sources found in other ERP and fMRI studies for late positivities and P300 component (Polich, 2007; Bledowski et al., 2004). The preponderance of a left distribution of SP generators could suggest access to a distributed system related to face identification as other studies have proposed (Barbeau et al., 2008; Joubert et al., 2006).
Finally, there was general consistency between the activations found in the imaging data of Experiment 1 and those modeled with the distributed inverse solution method employed in this study. fMRI, especially ROI analysis, and BMA found that conscious face perception correlates with the significant activation of left fusiform and inferior occipital areas as well as with the activation of parietal and frontal regions.

\section{GENERAL DISCUSSION}

In our study, conscious detection of face presentation was associated with higher activation of FFA and fronto-parietal areas as well as with larger N1 (N170), N2, and SP components. Modulations of early components of visual ERPs as well as of the level of activity in ventral stream areas have been found to correlate with conscious perception (Koivisto et al., 2008; Bar et al., 2001; Tong, Nakayama, Vaughan, \& Kanwisher, 1998). In our study, importantly, no region showed evidence for face-selective activity in the complete absence of awareness, indicated by reports of "no impression" of a face. Because the latter comparison-trials where faces are missed versus trials where subjects correctly reported there was no face-is crucial for the present study, absence of unconscious face processing cannot be explained by lack of design efficiency. We claim that our design is as efficient as we could reasonably have expected from the subject's responses.

The boundary conditions on this result remain to be determined. In a small handful of previous studies, fMRI or ERP evidence has suggested unconscious processing of faces or other objects, despite strong criteria for lack of awareness. These criteria include detailed trial-by-trial reports, as the present study (Sterzer et al., 2008), and evidence for chance performance in forced-choice presence/ absence testing (Jiang et al., 2009; Jiang \& He, 2006; Fang \& He, 2005; Williams, Liddell, et al., 2004). In the majority of these studies, notably, the method used to eliminate awareness was binocular suppression rather than backward masking. Plausibly, in the extended stimulus presentation times of binocular suppression studies, there is greater opportunity for consciously suppressed information to drive high-level object processing. A second possibility is that unconscious processing may be stronger for stimuli with a strong emotional significance (Jiang et al., 2009; Jiang \& He, 2006; Williams, Liddell, et al., 2004). Several findings suggest that stimuli with emotional content are indeed processed more efficiently that neutral stimuli (Zeelenberg, Wagenmakers, \& Rotteveel, 2006; Schupp, Junghofer, Weike, \& Hamm, 2003; Vuilleumier \& Schwartz, 2001). The mechanism responsible for the enhanced processing of emotional stimuli is not fully understood but may involve the amygdala and subcortical structures, including the reticular activating system and superior colliculus (Zeelenberg et al., 2006; Adolphs, 2004; Williams, Morris, McGlone, Abbott, \& Mattingley, 2004). 
Meanwhile, the results presented here point to the importance of a detailed assessment of awareness in studies examining activity related to unseen stimulus events. With less detailed assessment, trials wherein subjects have weak impressions of stimulus presence could be classified as misses, and resulting brain activity misinterpreted as unconscious (Hannula et al., 2005; Pessoa, 2005). With the power of our fMRI and ERP experiments, we found no evidence for face-related brain activity when faces were genuinely unperceived. These results suggest that a truly unperceived stimulus - at least under conditions of backward masking and perhaps excluding some stimuli with strong emotional content-registers very little in the brain, even in the earliest object-specific regions. Instead, face-related activity throughout the brain-both in ventral stream and frontoparietal cortex-is closely linked to conscious perception.

\section{APPENDIXES}

Appendix A. Experiment 1: Coordinates for Each Subject of the Center of Mass of FFA and OFA ROIs Defined Using Fusiform and Inferior Occipital AAL ROI as Search Spaces to Identify Significant Voxels $(p<.001$, Uncorrected) in the Contrast Face versus Scrambled Face of the Localizer Task

\begin{tabular}{|c|c|c|c|c|c|c|c|}
\hline \multirow[b]{2}{*}{ Subjects } & \multirow[b]{2}{*}{ Hemisphere } & \multicolumn{3}{|c|}{$F F A$} & \multicolumn{3}{|c|}{ OFA } \\
\hline & & $x$ & $y$ & $z$ & $x$ & $y$ & $z$ \\
\hline \multirow[t]{2}{*}{1} & $\mathrm{~L}$ & -36 & -51 & -20 & -41 & -78 & -8 \\
\hline & $\mathrm{R}$ & 38 & -51 & -18 & 43 & -76 & -9 \\
\hline \multirow[t]{2}{*}{2} & $\mathrm{~L}$ & -32 & -49 & -19 & & - & \\
\hline & $\mathrm{R}$ & 34 & -48 & -19 & 44 & -84 & -5 \\
\hline \multirow[t]{2}{*}{3} & $\mathrm{~L}$ & -36 & -55 & -16 & -42 & -77 & -9 \\
\hline & $\mathrm{R}$ & 39 & -49 & -20 & 41 & -80 & -8 \\
\hline \multirow[t]{2}{*}{4} & $\mathrm{~L}$ & & - & & -46 & -75 & -6 \\
\hline & $\mathrm{R}$ & 39 & -55 & -17 & 37 & -84 & -4 \\
\hline \multirow[t]{2}{*}{5} & $\mathrm{~L}$ & -40 & -55 & -19 & -41 & -80 & -7 \\
\hline & $\mathrm{R}$ & 40 & -46 & -20 & 43 & -77 & -6 \\
\hline \multirow[t]{2}{*}{6} & $\mathrm{~L}$ & -41 & -45 & -23 & & - & \\
\hline & $\mathrm{R}$ & 43 & -47 & -21 & 43 & -77 & -9 \\
\hline \multirow[t]{2}{*}{7} & $\mathrm{~L}$ & -40 & -56 & -18 & -39 & -82 & -10 \\
\hline & $\mathrm{R}$ & 37 & -50 & -17 & 40 & -81 & -8 \\
\hline \multirow[t]{2}{*}{8} & $\mathrm{~L}$ & -41 & -57 & -19 & -39 & -76 & -11 \\
\hline & $\mathrm{R}$ & 43 & -48 & -21 & 44 & -74 & -13 \\
\hline \multirow[t]{2}{*}{9} & $\mathrm{~L}$ & -37 & -54 & -14 & & - & \\
\hline & $\mathrm{R}$ & 40 & -41 & -18 & 45 & -74 & -9 \\
\hline \multirow[t]{2}{*}{10} & $\mathrm{~L}$ & & - & & -42 & -79 & -9 \\
\hline & $\mathrm{R}$ & 41 & -42 & -21 & 46 & -77 & -9 \\
\hline
\end{tabular}

Appendix A. (continued)

\begin{tabular}{|c|c|c|c|c|c|c|c|}
\hline \multirow[b]{2}{*}{ Subjects } & \multirow[b]{2}{*}{ Hemisphere } & \multicolumn{3}{|c|}{$F F A$} & \multicolumn{3}{|c|}{$O F A$} \\
\hline & & $x$ & $y$ & $z$ & $x$ & $y$ & $z$ \\
\hline \multirow[t]{2}{*}{11} & $\mathrm{~L}$ & -37 & -31 & -26 & & - & \\
\hline & $\mathrm{R}$ & 42 & -37 & -21 & & - & \\
\hline \multirow[t]{2}{*}{12} & $\mathrm{~L}$ & -35 & -47 & -18 & -43 & -77 & -6 \\
\hline & $\mathrm{R}$ & 39 & -44 & -18 & & - & \\
\hline \multirow[t]{2}{*}{13} & $\mathrm{~L}$ & -29 & -43 & -17 & -36 & -84 & -8 \\
\hline & $\mathrm{R}$ & 34 & -45 & -18 & 40 & -81 & -8 \\
\hline \multirow[t]{2}{*}{14} & $\mathrm{~L}$ & -34 & -52 & -18 & & - & \\
\hline & $\mathrm{R}$ & 36 & -50 & -18 & 45 & -75 & -6 \\
\hline \multirow[t]{2}{*}{15} & $\mathrm{~L}$ & -36 & -52 & -18 & -41 & -78 & -9 \\
\hline & $\mathrm{R}$ & 36 & -47 & -18 & 41 & -83 & -8 \\
\hline \multirow[t]{2}{*}{16} & $\mathrm{~L}$ & -40 & -61 & -14 & -39 & -73 & -11 \\
\hline & $\mathrm{R}$ & 40 & -52 & -17 & 41 & -77 & -7 \\
\hline \multirow[t]{2}{*}{17} & $\mathrm{~L}$ & -39 & -51 & -20 & -44 & -76 & -9 \\
\hline & $\mathrm{R}$ & 40 & -51 & -19 & 43 & -79 & -9 \\
\hline \multirow[t]{2}{*}{18} & $\mathrm{~L}$ & -39 & -58 & -17 & -41 & -78 & -8 \\
\hline & $\mathrm{R}$ & 38 & -50 & -18 & 42 & -76 & -10 \\
\hline
\end{tabular}

$\mathrm{R}=$ right; $\mathrm{L}=$ left.

Appendix B. Experiment 1: Total Numbers of Trials for Each Combination of Response and Exposure Time in Each Participant

For each stimulus (face or scrambled) and exposure time, trial numbers sum to (105 - [failures to respond]). In each participant, exposures selected for fMRI analysis are marked with *.

\begin{tabular}{lrrrrrr}
\hline Exposure & Hit & UF & Miss & CR & US & FA \\
\hline$S 1$ & & & & & & \\
$30 *$ & 9 & 24 & 72 & 78 & 17 & 10 \\
$50^{*}$ & 28 & 30 & 47 & 67 & 26 & 12 \\
67 & 62 & 21 & 21 & 59 & 31 & 15 \\
83 & 76 & 16 & 13 & 73 & 22 & 10 \\
52 & & & & & & \\
$30 *$ & & & & & & \\
$50 *$ & 36 & 21 & 48 & 70 & 21 & 14 \\
67 & 82 & 12 & 11 & 70 & 21 & 14 \\
83 & 98 & 0 & 7 & 68 & 27 & 9 \\
\hline
\end{tabular}


Appendix B. (continued)

\begin{tabular}{|c|c|c|c|c|c|c|c|c|c|c|c|c|c|}
\hline Exposure & Hit & $U F$ & Miss & $C R$ & $U S$ & $F A$ & Exposure & Hit & $U F$ & Miss & $C R$ & US & $F A$ \\
\hline S3 & & & & & & & S10 & & & & & & \\
\hline 30 & 0 & 6 & 99 & 97 & 7 & 0 & 30 & 0 & 15 & 89 & 91 & 12 & 1 \\
\hline $50 *$ & 28 & 15 & 61 & 93 & 11 & 0 & 50 & 2 & 35 & 67 & 77 & 24 & 1 \\
\hline $67 *$ & 70 & 19 & 16 & 80 & 23 & 1 & $67 *$ & 28 & 46 & 29 & 60 & 42 & 1 \\
\hline 83 & 89 & 8 & 6 & 69 & 31 & 4 & $83^{*}$ & 52 & 35 & 17 & 39 & 62 & 3 \\
\hline$S 4$ & & & & & & & $S 11$ & & & & & & \\
\hline 30 & 3 & 21 & 74 & 70 & 26 & 3 & $30^{*}$ & 14 & 22 & 61 & 66 & 30 & 4 \\
\hline $50 *$ & 8 & 42 & 47 & 66 & 29 & 4 & $50 *$ & 56 & 12 & 28 & 81 & 17 & 2 \\
\hline $67 *$ & 46 & 30 & 24 & 60 & 37 & 2 & 67 & 83 & 12 & 7 & 71 & 24 & 0 \\
\hline 83 & 74 & 18 & 8 & 60 & 38 & 2 & 83 & 83 & 8 & 6 & 82 & 19 & 0 \\
\hline$S 5$ & & & & & & & $S 12$ & & & & & & \\
\hline 30 & 4 & 19 & 82 & 96 & 9 & 0 & $30 *$ & 30 & 13 & 62 & 102 & 3 & 0 \\
\hline $50 *$ & 43 & 29 & 33 & 91 & 13 & 0 & $50^{*}$ & 82 & 9 & 14 & 98 & 6 & 1 \\
\hline $67 *$ & 77 & 13 & 15 & 92 & 12 & 1 & 67 & 101 & 2 & 2 & 96 & 8 & 1 \\
\hline 83 & 95 & 6 & 4 & 97 & 7 & 1 & 83 & 101 & 3 & 1 & 89 & 15 & 1 \\
\hline$S 6$ & & & & & & & $S 13$ & & & & & & \\
\hline $30 *$ & 10 & 22 & 73 & 89 & 15 & 1 & $30^{*}$ & 14 & 25 & 66 & 83 & 18 & 4 \\
\hline $50 *$ & 71 & 24 & 10 & 81 & 24 & 0 & $50^{*}$ & 63 & 15 & 27 & 80 & 21 & 4 \\
\hline 67 & 99 & 4 & 2 & 80 & 23 & 2 & 67 & 90 & 6 & 8 & 83 & 17 & 5 \\
\hline 83 & 101 & 3 & 1 & 83 & 20 & 2 & 83 & 98 & 4 & 3 & 86 & 17 & 1 \\
\hline$S 7$ & & & & & & & S14 & & & & & & \\
\hline 30 & 2 & 9 & 88 & 101 & 3 & 0 & $30^{*}$ & 17 & 25 & 63 & 73 & 30 & 1 \\
\hline $50 *$ & 45 & 27 & 29 & 92 & 8 & 0 & $50 *$ & 67 & 29 & 9 & 78 & 25 & 2 \\
\hline $67 *$ & 87 & 5 & 11 & 92 & 5 & 3 & 67 & 96 & 7 & 2 & 86 & 18 & 1 \\
\hline 83 & 97 & 4 & 3 & 92 & 7 & 0 & 83 & 99 & 4 & 2 & 90 & 14 & 1 \\
\hline$S 8$ & & & & & & & S15 & & & & & & \\
\hline 30 & 1 & 1 & 103 & 104 & 0 & 0 & $30^{*}$ & 6 & 53 & 46 & 78 & 26 & 1 \\
\hline $50 *$ & 16 & 0 & 89 & 102 & 0 & 1 & $50 *$ & 42 & 56 & 7 & 76 & 27 & 2 \\
\hline $67 *$ & 56 & 0 & 49 & 101 & 0 & 1 & 67 & 79 & 22 & 4 & 75 & 29 & 0 \\
\hline 83 & 74 & 0 & 31 & 95 & 0 & 6 & 83 & 92 & 12 & 1 & 81 & 23 & 1 \\
\hline S9 & & & & & & & $S 16$ & & & & & & \\
\hline $30 *$ & 16 & 43 & 46 & 74 & 30 & 1 & 30 & 2 & 35 & 65 & 82 & 20 & 3 \\
\hline $50 *$ & 71 & 25 & 8 & 80 & 22 & 3 & $50^{*}$ & 47 & 38 & 18 & 56 & 46 & 2 \\
\hline 67 & 91 & 12 & 2 & 79 & 25 & 1 & $67^{*}$ & 93 & 6 & 6 & 59 & 42 & 1 \\
\hline 83 & 101 & 3 & 1 & 87 & 16 & 2 & 83 & 99 & 3 & 3 & 59 & 44 & 2 \\
\hline
\end{tabular}

Appendix B. (continued)

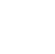


Appendix B. (continued)

\begin{tabular}{lrrrrrr}
\hline Exposure & Hit & UF & Miss & CR & US & FA \\
\hline S17 & 2 & 37 & 66 & 70 & 35 & 0 \\
30 & 13 & 46 & 46 & 45 & 57 & 2 \\
$50 *$ & 56 & 37 & 12 & 32 & 68 & 5 \\
$67 *$ & 84 & 20 & 2 & 21 & 78 & 6 \\
83 & & & & & & \\
518 & 10 & 9 & 86 & 73 & 20 & 9 \\
$30 *$ & 84 & 24 & 73 & 71 & 22 & 11 \\
$50 *$ & 37 & 35 & 33 & 47 & 41 & 17 \\
67 & 65 & 27 & 13 & 18 & 56 & 31 \\
\hline 83 & & & & & &
\end{tabular}

\section{Acknowledgments}

This work was funded by the Medical Research Council (UK) intramural program (U.1055.01.001.00001.01) and the UK/Cuba collaboration grants from the British Council and Royal Society. We would like to thank Eduardo Martínez and Lídice Galán for statistical advice and Nelson Trujillo for valuable comments on the manuscript.

Reprint requests should be sent to Valia Rodríguez, Cuban Neuroscience Center, Ave. 25 No.15202 esq. 158, Cubanacan, Playa, Havana 10400, Cuba, or via e-mail: valia.rodriguez@gmail.com.

\section{Note}

1. As an a priori criterion, we required at least six trials for the critical combinations of response category (Hit, Miss, CR) and exposure time, resulting in the elimination of data for 7 of 25 participants originally scanned. No behavioral or fMRI results from these additional participants are reported in this study.

\section{REFERENCES}

Adolphs, R. (2004). Emotional vision. Nature Neuroscience, 7, 1167-1168.

Allison, T., Puce, A., Spencer, D. D., \& McCarthy, G. (1999). Electrophysiological studies of human face perception. I: Potentials generated in occipitotemporal cortex by face and non-face stimuli. Cerebral Cortex, 9, 415-430.

Baars, B. J. (2002). The conscious access hypothesis: Origins and recent evidence. Trends in Cognitive Sciences, 6 , 47-52.

Bar, M., Tootell, R. B., Schacter, D. L., Greve, D. N., Fischl, B., Mendola, J. D., et al. (2001). Cortical mechanisms specific to explicit visual object recognition. Neuron, 29, 529-535.

Barbeau, E., Taylor, M. J., Regis, J., Marquis, P., Chanuvel, P., \& Liegeois-Chauvel, C. (2008). Spatio temporal dynamics of face recognition. Cerebral Cortex, 18, 997-1009.

Beck, D. M., Rees, G., Frith, C. D., \& Lavie, N. (2001). Neural correlates of change detection and change blindness. Nature Neuroscience, 4, 645-650.

Bentin, S., Allison, T., Puce, A., Perez, E., \& McCarthy, G. (1996).
Electrophysiological studies of face perception in humans. Journal of Cognitive Neuroscience, 8, 551-565.

Bentin, S., \& Deouell, L. Y. (2000). Structural encoding and identification in face processing: ERP evidence for separate mechanisms. Cognitive Neuropsychology, 17, 35-55.

Bledowski, C., Prvulovic, D., Goebel, R., Zanella, F. E., \& Linden, D. E. (2004). Attentional systems in target and distractor processing: A combined ERP and fMRI study. Neuroimage, 22, 530-540.

Brett, M., Anton, J. L., Valabregue, R., \& Poline, J. P. (2002). Region of interest analysis using an SPM toolbox. Neuroimage, 16, S497.

Buxton, R. B., Wong, E. C., \& Frank, L. R. (1998). Dynamics of blood flow and oxygenation changes during brain activation: The balloon model. Magnetic Resonance in Medicine, 39, 855-864.

Caharel, S., Fiori, N., Bernard, C., Lalonde, R., \& Rebai, M. (2006). The effects of inversion and eye displacements of familiar and unknown faces on early and late-stage ERPs. International Journal of Psychophysiology, 62, 141-151.

Carter, C. S., Braver, T. S., Barch, D. M., Botvinick, M. M., Noll, D., \& Cohen, J. D. (1998). Anterior cingulate cortex, error detection, and the online monitoring of performance. Science, 280, 747-749.

Collins, D. L., Zijdenbos, A. P., Kollokian, V., Sled, J. G., Kabani, N. J., Holmes, C. J., et al. (1998). Design and construction of a realistic digital brain phantom. IEEE Transactions on Medical Imaging, 17, 463-468.

Deffke, I., Sander, T., Heidenreich, J., Sommer, W., Curio, G., Trahms, L., et al. (2007). MEG/EEG sources of the 170-ms response to faces are co-localized in the fusiform gyrus. Neuroimage, 35, 1495-1501.

Dehaene, S., \& Naccache, L. (2001). Towards a cognitive neuroscience of consciousness: Basic evidence and a workspace framework. Cognition, 79, 1-37.

Dehaene, S., Naccache, L., Cohen, L., Bihan, D. L., Mangin, J. F., Poline, J. B., et al. (2001). Cerebral mechanisms of word masking and unconscious repetition priming. Nature Neuroscience, 4, 752-758.

Deichmann, R., Gottfried, J. A., Hutton, C., \& Turner, R. (2003). Optimized EPI for fMRI studies of the orbitofrontal cortex. Neuroimage, 19, 430-441.

Downar, J., Crawley, A. P., Mikulis, D. J., \& Davis, K. D. (2002). A cortical network sensitive to stimulus salience in a neutral behavioral context across multiple sensory modalities. Journal of Neurophysiology, 87, 615-620.

Dumontheil, I., Thompson, R., \& Duncan, J. (2011). Assembly and use of new task rules in fronto-parietal cortex. Journal of Cognitive Neuroscience, 23, 168-182.

Duncan, J. (2006). EPS Mid-Career Award 2004: Brain mechanisms of attention. Quarterly Journal of Experimental Psychology, 59, 2-27.

Duncan, J., \& Owen, A. M. (2000). Common regions of the human frontal lobe recruted by diverse cognitive demands. Trends in Neurosciences, 23, 475-483.

Eimer, M. (2000). Event-related brain potentials distinguish processing stages involved in face perception and recognition. Clinical Neurophysiology, 111, 694-705.

Fang, F., \& He, S. (2005). Cortical responses to invisible objects in the human dorsal and ventral pathways. Nature Neuroscience, 8, 1380-1385.

Friston, K. (1995). Neuronal transients. Proceedings of the Royal Society of London, Series B, Biological Sciences, 261, 401-405.

Furey, M. L., Tanskanen, T., Beauchamp, M. S., Avikainen, S., Uutela, K., Hari, R., et al. (2006). Dissociation of face-selective cortical responses by attention. Proceedings of the National Academy of Sciences, U.S.A., 103, 1065-1070. 
Grill-Spector, K., \& Kanwisher, N. (2005). Visual recognition: As soon as you know it is there, you know what it is. Psychological Science, 16, 152-160.

Halgren, E., Dale, A. M., Sereno, M. I., Tootell, R. B., Marinkovic, K., \& Rosen, B. R. (1999). Location of human face-selective cortex with respect to retinotopic areas. Human Brain Mapping, 7, 29-37.

Hampshire, A., Thompson, R., Duncan, J., \& Owen, A. M. (2008). The target selective neural response: Similarity, ambiguity, and learning effects. PLOS ONE, 3, e2520.

Hampshire, A., Thompson, R., Duncan, J., \& Owen, A. M. (2009). Selective tuning of the right inferior frontal gyrus during target detection. Cognitive, Affective, \& Behavioral Neuroscience, 9, 103-112.

Hannula, D. E., Simous, D. J., \& Cohen, N. J. (2005). Imaging implicit perception: Promise and pitfalls. Nature Reviews Neuroscience, 6, 247-255.

Haxby, J. V., Hoffman, E. A., \& Gobbini, M. I. (2000). The distributed human neural system for face perception. Trends in Cognitive Sciences, 4, 223-233.

Henson, R. N., Goshen-Gottstein, Y., Ganel, T., Otten, L. J., Quayle, A., \& Rugg, M. D. (2003). Electrophysiological and haemodynamic correlates of face perception, recognition and priming. Cerebral Cortex, 13, 793-805.

Henson, R. N., Mouchlianitis, E., Matthews, W. J., \& Kouider, S. (2008). Electrophysiological correlates of masked face priming. Neuroimage, 40, 884-895.

Herrmann, M. J., Ehlis, A. C., Muehlberger, A., \& Fallgatter, A. J. (2005). Source localization of early stages of face processing. Brain Topography, 18, 77-85.

Hoffman, E. A., \& Haxby, J. V. (2000). Distinct representations of eye gaze and identity in the distributed human neural system for face perception. Nature Neuroscience, 3, 80-84.

Ishai, A., Schmidt, C. F., \& Boesiger, P. (2005). Face perception is mediated by a distributed cortical network. Brain Research Bulletin, 67, 87-93.

Itier, R. J., \& Taylor, M. J. (2004). Source analysis of the N170 to faces and objects. NeuroReport, 15, 1261-1265.

Jeffreys, D. A. (1989). A face-responsive potential recorded from the human scalp. Experimental Brain Research, 78, 193-202.

Jeffreys, D. A. (1996). Evoked potential studies of face and object processing. Visual Cognition, 3, 1-38.

Jennings, J. R., \& Wood, C. C. (1976). The e-adjustment procedure for repeated-measure analyses of variance. Psychophysiology, 13, 277-278.

Jiang, Y., \& He, S. (2006). Cortical responses to invisible faces: Dissociating subsystems for facial-information processing. Current Biology, 16, 2023-2029.

Jiang, Y., Shannon, R. W., Vizueta, N., Bernat, E. M., Patrick, C. J., \& He, S. (2009). Dynamics of processing invisible faces in the brain: Automatic neural encoding of facial expression information. Neuroimage, 44, 1171-1177.

Joubert, S., Felician, O., Barbeau, E., Ranjeva, J. P., Christophe, M., Didic, M., et al. (2006). The right temporal lobe variant of frontotemporal dementia: Cognitive and neuroanatomical profile of three patients. Journal of Neurology, 253, 1447-1458.

Joyce, C., \& Rossion, B. (2005). The face-sensitive N170 and VPP components manifest the same brain processes: The effect of reference electrode site. Clinical Neurophysiology, 116, 2613-2631.

Kanwisher, N., McDermott, J., \& Chun, M. M. (1997). The fusiform face area: A module in human extrastriate cortex specialized for face perception. Journal of Neuroscience, 17, 4302-4311.

Kleinschmidt, A., Buchel, C., Zeki, S., \& Frackowiak, R. S. (1998). Human brain activity during spontaneously reversing perception of ambiguous figures. Proceedings of the Royal Society of London, Series B, Biological Sciences, 265, 2427-2433.

Koivisto, M., Lahteenmaki, M., Sorensen, T. A., Vangkilde, S., Overgaard, M., \& Revonsuo, A. (2008). The earliest electrophysiological correlate of visual awareness? Brain and Cognition, 66, 91-103.

Kouider, S., Eger, E., Dolan, R., \& Henson, R. N. (2009). Activity in face-responsive brain regions is modulated by invisible, attended faces: Evidence from masked priming. Cerebral Cortex, 19, 13-23.

Kranczioch, C., Debener, S., Schwarzbach, J., Goebel, R., \& Engel, A. K. (2005). Neural correlates of conscious perception in the attentional blink. Neuroimage, 24, 704-714.

Kriegeskorte, N., Goebel, R., \& Bandettini, P. (2006). Information-based functional brain mapping. Proceedings of the National Academy of Sciences, U.S.A., 103, 3863-3868.

Lamy, D., Salti, M., \& Bar-Haim, Y. (2009). Neural correlates of subjective awareness and unconscious processing: An ERP study. Journal of Cognitive Neuroscience, 21, 1435-1446.

Lau, H. C., \& Passingham, R. E. (2007). Unconscious activation of the cognitive control system in the human prefrontal cortex. Neuroscience, 27, 5805-5811.

Liu, J., Harris, A., \& Kanwisher, N. (2002). Stages of processing in face perception: An MEG study. Nature Neuroscience, 5, 910-916.

Lumer, E. D., Friston, K., \& Rees, G. (1998). Neural correlates of perceptual rivalry in the human brain. Science, 280 1930-1934.

MacDonald, A. W., Cohen, J. D., Stenger, V. A., \& Carter, C. S. (2000). Dissociating the role of the dorsolateral prefrontal and anterior cingulate cortex in cognitive control. Science, 288, 1835-1838

Marois, R., Do-Joon, L. Y., \& Marvin, C. M. (2004). The neural fate of consciously perceived and missed events in the attentional blink. Neuron, 41, 465-472.

Matsumoto, N., Okada, M., Sugase, Y., Yamane, S., \& Kawano, K. (2005). Population dynamics of face-responsive neurons in the inferior temporal cortex. Cerebral Cortex, 15, 1103-1112.

Miller, E. K., \& Cohen, J. D. (2001). An integrative theory of prefrontal cortex function. Annual Review of Neuroscience, 24, 167-202.

Mnatsakanian, E. V., \& Tarkka, I. M. (2004). Familiar-face recognition and comparison: Source analysis of scalp-recorded event-related potentials. Clinical Neurophysiology, 115, 880-886.

Moutoussis, K., \& Zeki, S. (2002). The relationship between cortical activation and perception investigated with invisible stimuli. Proceedings of the National Academy of Sciences, U.S.A., 99, 9527-9532.

Nasr, S., \& Esteky, H. (2009). A study of N250 event-related brain potential during face and non-face detection tasks. Journal of Vision, 9, 5-14.

Olivares, E. I., \& Iglesias, J. (2008). Brain potentials and integration of external and internal features into face representations. International Journal of Psychophysiology, 68, 59-69.

Pessoa, L. (2005). To what extent are emotional visual stimuli processed without attention and awareness? Current Opinion in Neurobiology, 15, 188-196.

Pessoa, L., \& Ungerleider, L. G. (2004). Neural correlates of change detection and change blindness in a working memory task. Cerebral Cortex, 14, 511-520.

Pins, D., \& Ffytche, D. (2003). The neural correlates of conscious vision. Cerebral Cortex, 13, 461-474.

Polich, J. (2007). Updating P300: An integrative theory of P3a and P3b. Clinical Neurophysiology, 118, 2128-2148. 
Rees, G., Kreiman, G., \& Koch, C. (2002). Neural correlates of consciousness in humans. Nature Reviews Neuroscience, 3, 261-270.

Rorden, C., \& Brett, M. (2000). Stereotaxic display of brain lesions. Behavioural Neurology, 12, 191-200.

Rossion, B., Joyce, C., Cottrell, G. W., \& Tarr, M. J. (2003). Early lateralization and orientation tuning for face, word, and object processing in the visual cortex. Neuroimage, 20, 1609-1624.

Rush, S., \& Driscoll, D. (1969). EEG electrode sensitivity-An application of reciprocity. IEEE Transactions on Biomedical Engineering, 16, 15-22.

Sadeh, B., Podlipsky, I., Zhdanov, A., \& Yovel, G. (2010). Event-related potential and functional MRI measures of face-selectivity are highly correlated: A simultaneous ERP-fMRI investigation. Human Brain Mapping, 31, 1490-1501.

Schupp, H. T., Junghofer, M., Weike, A. I., \& Hamm, A. O. (2003). Emotional facilitation of sensory processing in the visual cortex. Psychological Science, 14, 7-13.

Schweinberger, S. R., Pickering, E. C., Jentzsch, I., Burton, A. M., \& Kaufmann, J. M. (2002). Event-related brain potential evidence for a response of inferior temporal cortex to familiar face repetitions. Brain Research, Cognitive Brain Research, 14, 398-409.

Sergent, C., Baillet, S., \& Dehaene, S. (2005). Timing of the brain events underlying access to consciousness during the attentional blink. Nature Neuroscience, 8, 1391-1400.

Shapiro, K., Johnston, S. J., Vogels, W., Zaman, A., \& Roberts, N. (2007). Increased functional magnetic resonance imaging activity during nonconscious perception in the attentional blink. NeuroReport, 18, 341-345.

Sterzer, P., Haynes, J. D., \& Rees, G. (2008). Fine-scale activity patterns in high-level visual areas encode the category of invisible objects. Journal of Vision, 8, 10-12.

Sterzer, P., Jalkanen, L., \& Rees, G. (2009). Electromagnetic responses to invisible face stimuli during binocular suppression. Neuroimage, 46, 803-808.

Stokes, M., Thompson, R., Cusack, R., \& Duncan, J. (2009). Top-down activation of shape-specific population codes in visual cortex during mental imagery. Journal of Neuroscience, 29, 1565-1572.

Sugase, Y., Yamane, S., Ueno, S., \& Kawano, K. (1999). Global and fine information coded by single neurons in the temporal visual cortex. Nature, 400, 869-873.

Tanaka, J. W., Curran, T., Porterfield, A. L., \& Collins, D. (2006). Activation of preexisting and acquired face representations: The N250 event-related potential as an index of face familiarity. Journal of Cognitive Neuroscience, 18, 1488-1497.

Tong, F., Nakayama, K., Vaughan, J. T., \& Kanwisher, N. (1998). Binocular rivalry and visual awareness in human extrastriate cortex. Neuron, 21, 753-759.

Trujillo-Barreto, N. J., Aubert-Vazquez, E., \& Valdes-Sosa, P. A. (2004). Bayesian model averaging in EEG/MEG imaging. Neuroimage, 21, 1300-1319.

Tzourio-Mazoyer, N., Landeau, B., Papathanassiou, D., Crivello, F., Etard, O., Delcroix, N., et al. (2002). Automated anatomical labeling of activations in SPM using a macroscopic anatomical parcellation of the MNI MRI single-subject brain. Neuroimage, 15, 273-289.

Ullsperger, M., \& von Cramon, D. Y. (2001). Subprocesses of performance monitoring: A dissociation of error processing and response competition revealed by event-related fMRI and ERPs. Neuroimage, 14, 1387-1401.

Vogel, E. K., Luck, S. J., \& Shapiro, K. L. (1998). Electrophysiological evidence for a postperceptual locus of suppression during the attentional blink. Journal of Experimental Psychology, 24, 1656-1674.

Vuilleumier, P., \& Schwartz, S. (2001). Emotional facial expressions capture attention. Neurology, 56, 153-158.

Williams, L. M., Liddell, B. J., Rathjen, J., Brown, K. J., Gray, J., Phillips, M., et al. (2004). Mapping the time course of nonconscious and conscious perception of fear: An integration of central and peripheral measures. Human Brain Mapping, 21, 64-74.

Williams, M. A., Morris, A. P., McGlone, F., Abbott, D. F., \& Mattingley, J. B. (2004). Amygdala responses to fearful and happy facial expressions under conditions of binocular suppression. Journal of Neuroscience, 24 , 2898-2904.

Wolfe, J. M. (1999). Inattentional amnesia. In V. Coltheart (Ed.), Fleeting memories (pp. 71-94). Cambridge, MA: MIT Press.

Worsley, K. J., \& Friston, K. J. (1995). Analysis of fMRI time-series revisited-Again. Neuroimage, 2, 173-181.

Zeelenberg, R., Wagenmakers, E. J., \& Rotteveel, M. (2006). The impact of emotion on perception: Bias or enhanced processing? Psychological Science, 17, 287-291.

Zion-Golumbic, E., \& Bentin, S. (2007). Dissociated neural mechanisms for face detection and configural encoding: Evidence from N170 and induced gamma-band oscillation effects. Cerebral Cortex, 17, 1741-1749. 